\title{
Impacto de la reforma del sistema de seguridad social sobre la equidad en los servicios de salud en Colombia
}

\author{
The impact of social security reform \\ on health services equity in Colombia
}

Juan Eduardo Céspedes-Londoño ${ }^{1}$

Iván Jaramillo-Pérez 2

Ramón Abel Castaño-Yepes 3

\footnotetext{
1 Consultores Internacionales en Seguridad Social y Salud. Calle 72A, No. 00-44 E., Of. 302, Bogotá, Colombia. cinssa@latino.net.co 2 Escuela Superior de Administración Pública. Diagonal 40, no 4GA-37, Bogotá, Colombia. ijaramil@colomsat.net.co 3 London School of Hygiene and Tropical Medicine. Keppel St., London WCIE 7HT, UK. rcastano@post.harvard.edu
}

Abstract To evaluate the impact on access to, and use of, health services in Colombia's new national heal th insurance system, the authors compared two cross sections of the population: before (1993) and after (1997), with the approval of Act 100, creating the General System for Social Security in Health (SGSSS). Two equity indicators were assessed: concentration curves (CC) and concentration indices $(\mathrm{Cl})$, summarizing the distribution of access to health care and utilization of health care services provided by the SGSSS according to income deciles. Between 1993 and 1997 , the $\mathrm{Cl}$ for access to insurance hal ved from 0.34 to 0.17 ; si multaneously, coverage increased from $23 \%$ to $57 \%$, especially among the poorest segments of the population, where it increased from $3.7 \%$ to $43.7 \%$ as a result of subsidies provided by local governments. The $\mathrm{Cl}$ for utilization of heal th care services did not vary si gnificantly. Increased disease preval ence and utilization of services among the insured, due to biased selection of risks and moral hazards, were also documented. These findings suggest a positive impact by the Reform on inequalities in access to health care insurance; however, a similar effect on inequities in utilization of health services is not clear.

Key words Equity; Health Sector Reform; Health Services; Health System; Social Security Reform in Heal th Care

Resumo Para evaluar el impacto sobre la equidad en el acceso y en la utilización de servicios de salud del nuevo Sistema General de Seguridad Social en Salud Colombiano (SGSSS), se compararon dos cortes transversales de la población, antes (1993) y después (1997) de promulgada la Ley 100, de 1993, que lo creó, con respecto a dos indicadores de equidad: Ias curvas (CC) eíndices de concentración (IC) correspondientes a la distribución del aseguramiento y a la utilización de servicios. Entre 1993 y 1997, el IC en el aseguramiento se redujo a la mitad, del 0,34 al 0,17; simultáneamente, Ia cobertura del SGSSS se incrementó del 23\% al 57\%, sobretodo entre los segmentos más pobres de la población, donde pasó del 3,1\% al 43,7\%, como resultado de los subsidios otorgados por los gobiernos locales. Los IC en Ia utilización de servicios variaron muy poco. Adi cionalmente, se detectó una mayor prevalencia de enfermedad y utilización de servicios entre los asegurados, debido a sel ección sesgada de riesgos y riesgo moral. Estos resultados sugi eren un impacto positivo del SGSSS sobre las inequidades en el acceso al aseguramiento; sin embargo, no se evidenció un impacto claro sobrela equidad en la utilización final de servicios.

Palavras-chave Equidad; Reforma del Sector Salud; Servicios de Salud; Sistema de Salud; Reforma dela Seguridad Social en Salud 


\section{Introducción}

Durante la década pasada, Colombia inició una reforma radical de su antiguo Sistema $\mathrm{Na}$ cional de Salud (SNS), con una misión muy clara: garantizar la cobertura de las necesidades de atención en salud de la población, con eficiencia, calidad, equidad y solidaridad. Este proceso aún no se ha consolidado y fue acometido en dos etapas. Inicialmente, a partir de 1990, se fortaleció significativamente la descentralización del sistema público de prestación de servicios de salud, que se había iniciado en la década anterior. Luego, a partir de 1993, se inició la introducción de nuevo modelo de aseguramiento público, altamente descentralizado: el Sistema General de Seguridad Social en Salud (SGSSS).

El nuevo SGSSS fue diseñado siguiendo un modelo de competencia regulada, para cumplir cuatro funciones fundamentales, cada una de las cuales comprende procesos de producción propios y excluyentes, altamente diferenciados entre si, a saber: (1) financiar la demanda de atención en salud; (2) prestar los servicios de salud; (3) articular las dos funciones anteriores, a través de dos regímenes de aseguramiento, contributivo y subsidiado, que administran las Entidades Promotoras de Salud (EPS); y, (4) modular el comportamiento de todo el SGSSS (Bitrán \& Céspedes, 2001; Londoño \& Frenk, 1997).

El modelo de financiamiento adoptado por el SGSSS está orientado a movilizar recursos públicos hacia la demanda. Estos recursos se originan a partir de fuentes parafiscales y fiscales que son administradas a través de un fondo central: el Fondo de Solidaridad y Garantía (FOSYGA) y los Fondos Territoriales de Salud (FTS), respectivamente. El FOSYGA y los FTS le pagan a las EPS una prima fija (diseñada con base el método de tasación comunitaria y ajustada por riesgo), la Unidad de Pago Capitado (UPC), por la administración del Plan Obligatorio de Servicios (POS). La afiliación al SGSSS depende del nivel socioeconómico y de la capacidad de compra de las personas. Las personas formalmente empleadas y los trabajadores independientes, con capacidad suficiente, se afilian al Régimen Contributivo (RC) y aportan el $12 \%$ de su salario al SGSSS. Estos aportes son recaudados a través de las EPS por delegación del Sistema; Ias EPS, a su vez, mediante un proceso de cruce de cuentas, compensan con el FOSYGA la diferencia entre el monto recaudado y la U PC que el SGSSS les reconoce por cada individuo afiliado. Los individuos más pobres de la población, sin suficiente capacidad de compra, se afilian al Régimen Subsidiado (RS), luego de un proceso de focalización individual, administrado por los gobiernos locales, a través de un proceso conocido como Sistema de Selección de Beneficiarios (SISBEN); una vez focalizados y afiliados al RS, el Estado subsidia a estas personas el valor de la UPC.

Los servicios de salud son prestados a través de Instituciones Prestadoras de Servicios de Salud (IPS). Estas instituciones son responsables de prestar los servicios previstos en el POS, el cual comprende un paquete integral de servicios. Son de naturaleza pública o privada y compiten entre sí. Las IPS públicas, en particular, se están transformando en Empresas Sociales del Estado (ESE), con autonomía jurídica, administrativa y financiera, como parte del proceso de descentralización.

La articulación entre las funciones de financiamiento y de prestación de servicios se da a través de las EPS. Estas entidades, que también son de naturaleza pública o privada y compiten entre si, administran el aseguramiento, incluidos los procesos de afiliación; recaudo de las cotizaciones obligatorias; cobro de los subsidios que otorga el Gobierno; y manejo del POS con criterios de atención gerenciada.

Por último, la función de dirección y gobierno, o modulación, de todo el SGSSS se da a través de dos procesos críticos. El primero, consiste en la construcción de las políticas sectoriales, los instrumentos regulatorios correspondientes, y la evaluación de sus resultados. Las agencias principales responsables son el $\mathrm{Mi}$ nisterio de Salud (MS), con el apoyo del Consejo Nacional de Seguridad Social en Salud (CNSS), y el Ministerio de Hacienda ( $\mathrm{MH}$ ). Estas instituciones, conjuntamente, han producido más de 1.000 regulaciones que norman en detalle la organización y el funcionamiento del Sistema (Fernández, 1996); no obstante, hay falencias en el diseño de algunas, especial mente las relacionadas con el financiamiento y el aseguramiento (Frenk, 1988; Hsiao,1998; Reyes \& Céspedes, 1999). La evaluación de los resultados de la política, o control estratégico del SGSSS, también le corresponde al MS (por delegación del Presidente de la República), conjuntamente con el Consejo Nacional de Política Social y Económica (CONPES), y con el apoyo del Departamento Nacional de Planeación (DANE). El segundo proceso comprende la supervisión y el control del comportamiento de toda la base institucional del Sistema, de acuerdo con el marco de regulaciones y normas vigentes, responsabilidad que recae principal mente en la SNS.

Este diseño, sui generis, adoptado por el SGSSS colombiano resulta atractivo en su concepción teórica. Sin embargo, su implementa- 
ción enfrentó enormes desafíos para trascender de la letra del papel (representada en las leyes y sus reglamentaciones posteriores, contenidas en las más de 1.000 regulaciones y normas expedidas) a resultados concretos en relación con sus objetivos centrales.

Pero finalmente lo logró. De hecho, los efectos fueron sentidos en forma inmediata y, además, traumática por toda la base institucional del Sector (Restrepo, 1997). Por ejemplo, fue necesario liquidar la mayoría de las más de 1.000 cajas de previsión social que antes administraban la seguridad social; muchas EPS ineficientes, especialmente en el RS, quebraron o fueron liquidadas; algunos de los hospitales más grandes y tradicionales del país, de origen público o solidario, también quebraron debido a problemas de eficiencia y calidad; y una fracción importante del gasto social, en general, se fugó como consecuencia de la corrupción rampante que la descentralización generó en muchos gobiernos locales, de acuerdo con la Contraloría General de la Nación y la Defensoría del Pueblo (Málaga et al., 2000).

Esta situación se explica porque la implementación del nuevo SGSSS modificó en forma radical el antiguo SNS, sin una adecuada asistencia técnica por parte del Gobierno, y produjo dos reacciones importantes. Primero, insatisfacción e intensa oposición por parte de algunos actores, especialmente los profesionales de la salud, quienes critican las consecuencias adversas del modelo de atención gerenciada que subyace al POS, sobre sus ingresos y la calidad técnica de los servicios de salud (Patiño, 1999). Segundo, un acalorado debate y controversia en relación con su validez y viabilidad a largo plazo, en un ambiente politizado y cargado ideológicamente (Ahumada, 1999; ALAMES, 2000; Franco, 2000; Hernández, 2000).

De otro lado, el impacto de la Reforma sobre la población fue aún más significativo. Durante 1993 y 1997, la cobertura del aseguramiento pasó del 20 al 57 por ciento de la población. Este resultado se debió a dos factores fundamentales: (1) extensión de la cobertura a los dependientes de los individuos que cotizaban a una de más de 1000 instituciones de seguridad que existían con anterioridad a 1993; y, (2) el otorgamiento de subsidios a cerca de 7,0 millones de colombianos, que posibilitó su afiliación al SGSSS (Hernández, 2000; Reyes et al., 1998). Adicionalmente, cerca del 85 por ciento de la población está satisfecha con los servicios de salud que recibe (Reyes et al., 1998), y la calificación promedio que los colombianos hacen de los servicios de salud es superior a 4,0, en una escala de 1 a 5 (Giraldo, 2000).
En cambio, los resultados observados en relación con la eficiencia son cuestionables, porque el desempeño del aseguramiento debería haber sido mayor frente a un gasto total en salud que pasó del 7 al 10 por ciento del Producto Interno Bruto (PIB) durante el mismo período (Céspedes, 1998a; Morales, 1997; Ruiz \& O'Meara, 1999).

Los posibles efectos de la Reforma sobre la equidad en la prestación de los servicios de salud se desconocían con anterioridad a la presente investigación. Específicamente, este estudio se justificó sobre la base de dos interrogantes planteados: ¿Cuáles fueron los resultados concretos obtenidos por la instrumentación y posterior implementación de las políticas regulatorias que originaron el nuevo SGSSS, sobre las siguientes dimensiones de la equidad en la prestación de los servicios de salud: (1) acceso de la población a través del aseguramiento y, (2) accesibilidad y utilización final de las instituciones prestadoras de servicios de salud? Las respuestas a estos interrogantes constituyen un insumo necesario para que los planificadores y formuladores de políticas públicas puedan perfeccionar y mejorar la eficacia de los instrumentos de regulación del SGSSS, especialmente aquellos destinados a focalizar el gasto social hacia los segmentos más pobres, marginados y vulnerables de la población, con el fin de garantizar el acceso al aseguramiento y la accesibilidad a los servicios de salud (Gwatkin, 2001; Hernández \& Zapata, 1991; Neufeld \&Johnson, 2000; PAHO/WHO, 2001; Ramalingaswami, 1993).

El presente artículo consta de cuatros partes. En la primera parte, se describen los principales antecedentes de política social y económica que enmarcaron la reforma del SGSSS colombiano, como los argumentos sobre equidad que sirvieron de soporte a la presente investigación. En la segunda, se presentan el marco conceptual y la metodología. Y, en la tercera, se analizan las evidencias relacionadas con los resultados obtenidos por el SGSSS sobre la equidad en el acceso y la accesibilidad final a los servicios de salud por parte de la población.

\section{Reforma de la seguridad social}

El proceso de reforma de salud colombiano fue el resultado de un largo y complejo proceso de construcción de política sectorial. Este proceso se dio dentro de un contexto más amplio, el cual transformó radicalmente todo el modelo de desarrollo económico y social del país, en general. Las políticas de equidad, particularmen- 
te, se orientaron a crear condiciones de igualdad de oportunidades, tanto en el acceso como en la utilización de los servicios de salud, independientes del nivel socioeconómico y de la capacidad de compra de las personas.

La reforma fue adoptada formalmente a través de varios instrumentos claves. Primero, la Constitución de 1991 - creada por la Asamblea Nacional Constituyente (ANC), conformada en 1990 -, en la cual se consagraron los principios de eficiencia, calidad y equidad que enmarcaron el nuevo modelo de desarrollo económico y social, en general, y de seguridad social, en particular. Y, segundo, la Ley 100, de 1993 (CoIombia, 1993b; Patiño, 1999), promulgada por el Congreso de la República. Esta ley desarrolló estos principios constitucionales y definió formalmente la organización y el funcionamiento del nuevo SGSSS. Para ello, se apoyó en una Comisión Accidental de Seguridad Social, creada durante 1992, con el doble propósito de recoger y analizar evidencias teóricas y empíricas y de desarrollar un modelo concertado que incorporara las posiciones planteadas inicialmente desde la ANC. La ley dio origen a otros dos sistemas adicionales al SGSSS: Sistema General de Riesgos Profesionales (SGRP) y Sistema General de Pensiones (SGP). En particular, el SGSSS fue diseñado como un modelo de competencia regulada por el Estado. Adicionalmente, reconoció la intervención de la comunidad (a través de los beneficiarios del Sistema) sobre la "organización, control, gestión, fiscalización de las instituciones que integran el Sistema en su conjunto", en desarrollo del principio de "participación” (Isaacs, 2000). Este último principio corrobora el hecho de que el éxito del SGSSS depende de su comportamiento potencial como sistema auto-referente, es decir, bajo el control directo de sus beneficiarios y usuarios, y no exclusivamente del Estado.

Hay otras normas, que también afectan la organización y el funcionamiento del SGSSS. Las principales son las leyes 10, de 1990 (Colombia, 1990), y 60, de 1993 (Colombia, 1993a), que fortalecieron el proceso de descentralización de la red pública de instituciones prestadoras de servicios de salud; y las leyes 608 (Colombia, 1999b) y 489 (Colombia, 1999a), de 1999, que reformaron toda la administración pública.

El diseño resultante del SGSSS se asemeja conceptualmente a un modelo de Pluralismo Estructurado (Londoño \& Frenk, 1997). Su característica fundamental es que las funciones principales del Sistema están separadas e integradas horizontalmente. Algunas son responsabilidad directa del Estado (ej., dirección y gobierno, financiamiento, provisión de servicios de promoción y prevención, otorgamiento de subsidios). Otras son desarrolladas por agentes públicos y privados (ej., aseguramiento y prestación de servicios de salud). Este nuevo arreglo también se encuentra en otras reformas que están siendo introducidas por otras democracias pluralistas de la Región (Almeida, 1999; BID, 1995), y contrasta con el antiguo SNS, en donde coexistían varios sistemas independientes que integraban verticalmente las funciones de modulación, financiamiento y prestación de servicios; adicionalmente, representa la respuesta por parte de las autoridades de planeación económica y social (Jaramillo, 1999) a la ineficiencia, mala calidad e inequidad que caracterizaban al antiguo SNS (Yepes, 1999).

En general, la reforma de salud colombiana resulta atractiva porque las políticas e instrumentos que la enmarcaron se consideran bien enfocados frente a las necesidades y demandas prioritarias de salud de la población; y, además, es relevante e innovadora en relación con los demás objetivos centrales de eficiencia y calidad que orientan la respuesta organizada en salud y están consagrados en la Constitución. Este argumento es ampliamente aceptado, inclusive por parte de los más vigorosos contradictores (Patiño, 1999), y se apoya en tres hechos concretos. Primero, la Reforma colombiana responde a las nuevas reali dades y retos impuestos por la transición en salud que experimenta el país: la evolución acelerada hacia un perfil de necesidades, dominado por una carga creciente de enfermedades crónicas y traumas, que ejerce una presión, cada vez mayor, sobre la demanda y la utilización de tecnologías sofisticadas y costosas (Céspedes et al., 1995; Jamison et al., 1993; Murray, 1996). Segundo, el diseño adoptado está acorde con las teorías, experiencias y tendencias modernas sobre reforma de los sistemas de salud (Frenk, 1988; Harvard University, 1993; Hsiao, 1998; Jack, 1999; Marset-Campos et al., 1998; WB, 1993, 1997, 2001). Tercero, es coherente con las opiniones y recomendaciones de muchos expertos en regulación y reforma de sistemas de salud (Harvard University, 1996; Preker et al., 1999).

Además, retó paradigmas tradicionales en salud pública (Chernichovsky, 1995). Esto generó intensa controversia y debate sobre su validez y viabilidad, como se vió. Los grupos que, de alguna manera, han visto afectados sus intereses especiales, como los profesionales de la salud, los hospitales y los sindicatos, han sido particularmente críticos y reticentes al cambio. Es más, en la práctica, la Reforma tuvo consecuencias iniciales muy traumáticas sobre la base institucional del Sector, debido a la forma 
abrupta y radical como se dio el proceso de cambio, sumado a la poca asistencia técnica por parte del Gobierno para apoyarlo. Estos traumatismos (aunque en cierta medida inevitables, principalmente por lo radical del cambio) acrecentaron aún más la controversia en torno a la validez del SGSSS como instrumento fundamental de la política de salud colombiana.

Por estas razones, la evolución del SGSSS no corresponde con precisión a un modelo teórico "puro", sino más bien a la historia de adaptaciones que debió atravesar para nacer y sobrevivir (Bitrán \& Cespedes, 2001; Yepes, 2000). Los inevitables avatares de los procesos políticos lo obligaron a adaptarse desde su concepción a las diversas corrientes de pensamiento, fuerzas e intereses en juego.

Hoy día, el principal desafió que enfrenta la Reforma consiste en cómo mejorar la calidad de los instrumentos de política que afectan el diseño del SGSSS y la capacidad por parte del Estado para supervisar y controlar, o fiscalizar, su cumplimiento.

En relación con el diseño, el proceso de instrumentación no ha concluido aún. En algunos dominios de política regulatoria, tales como la prestación de servicios de salud, existen demasiadas regulaciones y normas frente a procesos críticos que podrían ser abordados con mayor eficacia a través de mecanismos de control intrínsecos o privados (ej., acreditación y control de la calidad, a través de asociaciones y empresas especializadas); en cambio, hay enormes vacíos frente a otros procesos que requieren controles estatales más estrictos (ej., licenciamiento). En el aseguramiento, las reglas de juego para administradoras del régimen subsidiado son insuficientes frente a procesos críticos tales como la contratación y las protecciones contra la insolvencia (ej., márgenes de solvencia, reservas técnicas, etc), orientados a crear condiciones de credibilidad y confianza en el Sistema. En otros dominios, tales como el financiamiento, las normas no están contenidas en un solo estatuto y están dispersas en varias regulaciones, lo cual dificulta su comprensión e, inclusive, genera conflictos y colisiones. Más grave aún, no hay estabilidad suficiente en las normas, que son modificadas continuamente; ni imparcialidad (ej., en muchos casos, la reglas de juego son unas para empresas públicas y otras para las privadas), lo cual limita la credibilidad de las agencias reguladoras, de un lado, y la confianza de los agentes del mercado, del otro.

Pero, finalmente, la supervisión y el control de las regulaciones y normas resultan en proceso todavía más crítico, para garantizar la efectividad de un modelo de competencia regulada como el adoptado por Colombia. Sin embargo, en la práctica, las instituciones responsables presentan enormes falencias que amenazan con convertir la ley en letra muerta (Bitrán \& Céspedes, 2001).

\section{Marco conceptual y metodología}

Definiciones conceptuales de equidad

En relación con la equidad, los enfoques teóricos y conceptual es existentes establecen diferencias claras entre la equidad en las condiciones y necesidades de salud de una población, de un lado, y en la prestación de los servicios de salud, para responder a dichas necesidades, del otro lado.

En general, los conceptos sobre equidad en salud y en la prestación de los servicios se originan en principios éticos y valores sociales firmemente arraigados en las sociedades occidentaIes (Daniels, 1996; Folland et al., 1993; Hernández et al., 1995; Sen, 1992, 1999; Whitehead, 1992). Como consecuencia (y a diferencia de otros mercados donde la demanda de bienes y servicios varía en función de las necesidades y de la capacidad de compra de las personas), la salud es considerada como un bien meritorio en muchas sociedades, incluida la colombiana (Castaño, 1999; Sen, 1987); esta condición origina derechos fundamentales en las personas y deberes por parte de la sociedad, consagrados en la constitución y en las leyes que garantizan el acceso a los servicios de salud.

En desarrollo de dichos principios y valores, Ias teorías sobre equidad en salud parten de un hecho fundamental: entre los diferentes segmentos de las poblaciones (definidos en función de sus características demográficas, económicas, sociales y geográficas), es común observar desigualdades en los indicadores de salud (esperanza de vida, morbilidad, mortalidad), sobretodo al comparar los segmentos más pobres y vulnerables de las poblaciones con los demás segmentos más afortunados. Estas desigual dades entre segmentos de la población van en contravía de principios de justicia social y se originan en diferencias de orden biológico, ambiental, social, como en las condiciones de acceso, utilización e impacto de los servicios de salud (Daniels, 1996).

Sin embargo, con excepción de las diferencias de tipo biológico, los demás factores que inciden sobre las condiciones de salud de una población pueden ser intervenidos a través de políticas de salud y de desarrollo social y económico, en general. Por consiguiente, la equi- 
dad en salud se refiere a aquellas diferencias en los indicadores de salud que son evitables e injustas (Whitehead, 1992). En este sentido, se entiende como la igualdad de oportunidades que deben tener todas las personas para alcanzar su pleno potencial de salud sin desventaja, si ello puede evitarse; esto es distinto de la equidad en la prestación de los servicios de salud, que se define a partir de tres premisas: igual acceso a los servicios de salud para igual necesidad, igual uso de servicios para igual necesidad e igual calidad de la atención para todos, como se verá más adelante (Hernández et al., 1995).

Los conceptos sobre equidad discutidos arriba tienen implicaciones metodológicas sobre la medición e interpretación de los indicadores de salud y necesidades en poblaciones humanas (Hernández et al., 1995; Townsend \& Davidson, 1982)

Esto implica, por una parte, que los sistemas de salud deben ser financiados con esquemas tributarios progresivos, o al menos proporcionales, es decir, bien sea mediante impuestos generales directos bajo esquemas progresivos, o mediante impuestos a la nómina que eviten la transferencia del impuesto al trabajador o a los precios de los demás bienes y servicios de la economía (Kakwani, 1977). Por esta razón, los seguros privados y los pagos directos de bolsillo no deben constituir un esquema de financiamiento predominante, pues a su impacto regresivo hay que agregarle el impacto mucho más inequitativo que afecta en mayor medida a las personas enfermas (Hsiao \& Liu, 1996).

Por otra parte, la distribución equitativa de los servicios de salud implica una definición del concepto de necesidad, el cual debería orientar la distribución de los recursos. Esta definición plantea profundos cuestionamientos sobre cómo definir la necesidad; un individuo que presenta una enfermedad terminal, ciertamente necesita mucho más salud que cualquier otro, sin embargo, no es posi ble lograrlo; esto nos lleva a definir la necesidad como capacidad de beneficiarse, concepto que nos obliga a defender un valor intrínseco indiscutible que sea digno de perseguir, a costa de incurrir en un costo de oportunidad medido en otros tipos de beneficios, no solamente en materia de salud; en una esfera más elaborada, podría definirse la necesidad como la cantidad de recursos requeridos para agotar la capacidad de beneficiarse, es decir, que, entre dos individuos con la misma capacidad de beneficiarse de las intervenciones en salud, necesitaría más aquél que requiera más recursos para agotar dicha capacidad de beneficiarse (Culyer \& Wagstaff, 1993).
Otro criterio que se utiliza para analizar la equidad en la prestación de servicios es el acceso a los servicios de salud (Culyer \& Wagstaff, 1993; van Doorslaer et al., 1993); se dice que un sistema de salud es equitativo si todas las personas tienen igual oportunidad de obtener servicios de salud cuando así lo requieran, sin importar su capacidad de pago; la igual utilización de servicios de salud entre diferentes niveles de ingreso así como el establecimiento de iguales costos para todos encierran una sobre simplificación que desconoce preferencias individuales; de otra parte, al enfrentarse a los patrones de consumo de otros bienes y servicios, el individuo de menos ingresos tendrá siempre que renunciar a consumir más de estos bienes que el de más altos ingresos, lo cual nos lleva a concluir que, aún con precios iguales o acceso igual, existe un costo de oportunidad en términos de utilidad no percibida, que es mayor para el de menos ingresos.

En toda esta discusión es necesario recalcar la contraposición entre el concepto de equidad y el de eficiencia, pues la mejor distribución de los recursos para lograr el mayor beneficio posible implica inevitablemente restringir el acceso de algunos individuos, con grandes necesidades presentes, a los servicios de salud que requieren para resolver su situación (Whitehead, 1992). De igual manera, la medición los conceptos aquí enunciados ponen de manifiesto enormes retos metodológicos, que dificultan la realización objetiva del principio de equidad en la prestación de los servicios de salud.

\section{Diseño analítico}

La evaluación del comportamiento de los indicadores de equidad en la prestación de los servicios de salud se orientó hacia dos objetivos analíticos. Primero, analizar los resultados obtenidos por la Reforma sobre el aseguramiento, como mecanismo principal para garantizar el acceso a los servicios de salud; para ello, se compararon dos cortes transversales de la población, antes (1993) y después (1997) de promulgada la Ley 100, de 1993 (Colombia, 1993b), que creó el SGSSS, con respecto al comportamiento observado en la cobertura del aseguramiento según deciles ó cuartiles de ingreso de los hogares. Y, segundo, analizar los resultados obtenidos por el aseguramiento sobre la prestación de servicios de salud a la población; para ello, en cada uno de los dos cortes mencionados, se compararon dos grupos de población, afiliados y no afiliados a una empresa de aseguramiento, con respecto al comporta- 
miento de indicadores de equidad en la accesibilidad y utilización final de los servicios de salud, también según deciles o cuartiles de ingreso de los hogares.

Indicadores de equidad

y definiciones operacionales

En la construcción de los indicadores de equidad, considerados por la presente investigación, se tuvieron en cuenta dos dimensiones del SGSSS de Colombia: (1) las desigualdades en las condiciones y necesidades de salud de la población; y, (2) las inequidades en la prestación de los servicios de salud, en respuesta a dichas necesi dades. Para medir estas dos dimensiones del SGSSS, se adoptaron las definiciones operacionales que se describen a continuación.

Desigual dades en las condi ciones y necesidades de salud. Se expresan como variaciones en la distribución de los indicadores de salud, las cuales se manifiestan al comparar diferentes segmentos de la población con respecto a sus características principales: (1) demográficas (ej., edad y género); (2) geográficas (ej., región, procedencia: urbana o rural); (3) sociales (ej., ocupación, nivel de educación); (4) económicas (ej., deciles o cuartiles de ingreso del hogar); $y,(5)$ estado de afiliación a SGSSS, entre otras características. Como indicadores proxy de necesidades, se calcularon tres tasas de prevalencia en la población general: (1) enfermedad durante las últimas cuatro semanas, definida como la ocurrencia de al guna enfermedad, accidente, problema dental o problema de salud; (2) enfermedad con restricción de las actividades normales, definida como el haber estado restringido durante al menos un día en las actividades de la vida diaria, como consecuencia de la enfermedad, accidente o problema de salud durante el mes previo a la encuesta; y (3) estado de salud en general, definido como la percepción del estado de salud en general (excelente, bueno, regular o malo); estas medidas reflejan los modelos médico, funcional y subjetivo de salud, respectivamente, y fueron calculadas con el propósito de ser incluidas como variables de ajuste en el análisis de las inequidades en la prestación de los servicios de salud.

Inequidades en la prestación de los servicios desalud. Se expresan como desigualdades en la prestación de los servicios de salud, las cuales se manifiestan al efectuar comparaciones de la población según el tipo de afiliación a una empresa aseguradora, niveles de ingreso del hogar y otras características socio-económicas. Para evaluar este tipo de desigualdades, se construyeron los siguientes indicadores:
Inequidades en el acceso a los servicios de salud: se utilizaron dos medidas proxy para evaluar los efectos del SGSSS sobre los costos directos eindirectos. Para evaluar los efectos del aseguramiento sobre los costos directos relacionados con el acceso a los servicios de salud, se utilizó la tasa de prevalencia de cobertura en el aseguramiento, definida como el porcentaje de personas afiliadas a una empresa de aseguramiento (pública o privada), durante los dos períodos de tiempo considerados (1993 y 1997), en relación con la población total correspondiente a los mismos dos períodos de tiempo. Esta primera medida es considerada como un indicador de la efectividad de las políticas de financiamiento y aseguramiento adoptadas, con el propósito de reducir las barreras económicas que dificultan el acceso de la población, es decir, minimizar los costos directos para el usuario. Para evaluar los efectos del SGSSS sobre los costos indirectos del acceso a los servicios de salud, se analizaron las prevalencias, ajustadas por edad, género y actividad restringida, correspondientes a las razones para no utilizar los servicios de salud durante 1997. Estas medidas se definieron como los porcentajes de personas que no utilizaron los servicios de salud por causas determinadas, en relación con la población total con percepción de enfermedad durante el mismo período de tiempo. Entre las razones identificadas por las personas con percepción de enfermedad para no utilizar los servicios de salud, se incluyeron las siguientes: (a) caso leve, (b) no tuvo tiempo, (c) centro de atención lejos, (d) falta de dinero, (e) considera malo el servicio, ( $f$ ) no lo atendieron, (g) no confía en los médicos, (h) ha consultado antes y no resuelveron el problema, y (i) muchos trámites para la cita.

Inequidades en la utilización final de los servicios de salud: Para evaluar este tipo de inequidades, se construyeron dos indicadores: (1) probabilidad ajustada de utilización de servicios hospitalarios en el último año; y (2) probabilidad ajustada de utilización de servicios de salud ambulatorios en los últimos 30 días; este último indicador refleja principalmente la demanda efectiva de servicios extra-hospitalarios y ambulatorios. En el ajuste hecho por regresión logística a las probabilidades de uso de servicios, se incorporaron características biológicas de la población que están asociadas con la demanda y utilización de servicios de salud (ej., edad, género y necesidades de salud). Por tanto, al efectuar comparaciones entre distintos segmentos de la población (definidos según estado de afiliación al SGSSS y niveles de ingreso), la persistencia de diferencias en el compor- 
tamiento de los indicadores de utilización (no atribuibles a errores de muestreo) representa evidencia de inequidad en la accesibilidad final a los servicios de salud.

\section{Fuentes de datos e información}

Para construir los indicadores mencionados, se procesaron y analizaron las bases de datos correspondientes a dos encuestas socio-económicas, muy similares entre si en su diseño: (1) la Encuesta Nacional de Caracterización Socioeconómica (Casen), realizada por el Departamento Nacional de Planeación (DNP), durante 1993; y (2) la Encuesta de Calidad de Vida (Encalivida), realizada durante 1997, por el Departamento Administrativo Nacional de Estadísticas (DANE). En ambas encuestas, los datos e información fueron obtenidos por entrevista estructurada a las personas de los hogares, seleccionados a través de muestras complejas de las siguientes características: (1) probabilísticas, porque cada unidad de observación tiene una probabilidad superior a cero de ser incluida en la muestra; (2) de conglomerados, constituidos por los municipios en los diferentes municipios del país o unidades primarias de muestreo (UPM ), y los hogares y personas ó unidades secundarias de muestreo (USM); (3) estratificada por regiones del país y ubicación urbano-rural de los municipios y hogares; y (4) bietápica, porque las muestras fueron seleccionadas en dos etapas, primero las UPM y, luego, los elementos de observación dentro de las UPM.

Procesamiento y análisis

estadístico de los datos

El procesamiento y el análisis estadístico de los datos se hicieron utilizando los programas SPSS (SPSS Incorporation, 1997) y STATA (Stata Corporation, 1999). Primero, se efectuó un análisis exploratorio de los datos, para calcular las medidas de tendencia central de las variables originales, incluidas proporciones, medianas, promedios y modas. A continuación, se procedió a la recodificación y a la creación de nuevas variables; de esta forma, las variables continuas fueron recodificadas a variables discretas categóricas. Luego, se hicieron análisis bivariados, utilizando tablas de contingencia para el caso de variables discretas y boxpl ots para el caso de variables continuas, con el fin de hacer una evaluación inicial de las relaciones entre las variables dependientes (indicadores de salud y equidad) e independientes (característi- cas sociales, económicas y demográficas de la población). El cálculo de las medidas descriptivas se hizo con base en la muestra expandida.

Posteriormente, se hizo un ajuste de los indicadores de salud y de equidad, por el método directo (Wagstaff \& van Doorslaer, 1998; Wagstaff et al., 1999). Los indicadores de salud se ajustaron por edad y género. Lo indicadores de acceso y accesibilidad a los servicios de salud se ajustaron por edad, género y necesidades de salud, utilizando como proxy la ocurrencia de actividad restringinda. Para ello, se empleó el siguiente modelo de regresión logística (Hosmer \& Lameshow, 1989):

$$
Y=B o+B i X i+B j X j+B p X p+B i * B p
$$

donde $Y$ es la probabilidad de que el indicador sea 1 ó 0 , la cual resulta de la combinación lineal de los siguientes parámetros: (1) Bo, la constante; (2) Bi, las variables tenidas en cuenta en el ajuste (edad, género y otras); (3) Bj, las características socio-económicas de la población (cuartiles o deciles de ingreso del hogar); (4) $B p$, el estatus de afiliación a la seguridad social en salud ( $\mathrm{si}=1$ o no =0); y, (5) Bi*Bp, los términos de interacción. La probabilidad, o tasa ajustada, para cada grupo de población correspondiente al nivel socioeconómico y al estatus de afiliación, $p$, está dada por la diferencia de los parámetros entre $Y(p=1)$ y $Y(p=0)$, y se expresa en la siguiente ecuación:

$$
p(y)=\frac{1}{1+e^{-(B i X i+B j X j+B p X p+B j * B p)}}
$$

Las diferencias encontradas también se expresaron como razones de disparidad u odds ratios (OR), asociados con el aseguramiento o nivel socioeconómico (deciles de ingreso). Como categoría de referencia para el cálculo de Ios OR, se utilizó el primer decil de ingresos y, en el caso de variables dicótomas, también los valores codificados como cero. El cálculo de los OR y de los valores de significancia estadística correspondientes se hizo con base en la muestra expandida, incorporando a su cálculo las probabilidades desiguales utilizadas en la selección de las UPM.

Finalmente, se analizaron las distribuciones de los indicadores de acceso y utilización de servicios de salud, ajustados según estatus socioeconómico (deciles de ingreso de los hogares). Para ello, se adaptaron técnicas econométricas empleadas en la construcción de la Curva de Lorenz y el Coeficiente de Gini, que permitieron evaluar la distribución del gasto. De esta forma, se construyeron curvas para eva- 
luar la distribución de los servicios, L(s), y calcular los indicadores correspondientes de concentración de servicios, C, adaptando técnicas utilizadas originalmente para evaluar el gasto en salud (Wagstaff \& van Doorslaer, 1998). De esta forma, en un gráfico de dispersión, la curva $\mathrm{L}(\mathrm{s})$ representa la proporción acumulada de la población, por niveles socioeconómicos (deciles de ingreso del hogar de nuestro caso), contra la proporción acumulada de servicios utilizados. Si las dos distribuciones coinciden perfectamente, su relación se expresa como una línea recta diagonal (nivel de referencia); esto significa que no hay desigualdades en la distribución de los servicios que sean atribuibles a diferencias en el ingreso y, por consiguiente, que no hay inequidades en tal sentido. Pero, si la curva está por encima o debajo de la diagonal, ello indica inequidades a favor ó en contra de los segmentos más pobres, respectivamente (Wagstaff \& van Doorslaer, 1998).

El área debajo de la curva con respecto a la diagonal indica el grado de concentración de servicios y se puede evaluar por medio del índice de concentración, C, definido como el doble del área comprendida entre Ls y la diagonal.

$$
I=1-2 \int_{0}^{1} L(s) d s=C
$$

El índice de concentración toma el valor de cero cuando L(s) coincide con la diagonal, es negativo ó positivo, cuando L(s) está por encima o debajo de la diagonal, respectivamente.

La fórmula computacional para calcular C está dada por:

$$
\frac{2}{u} \sum_{t=1}^{T} f_{t} u_{t} R_{t}-1
$$

donde:

$$
u=\sum_{t=1}^{T} f_{t} u_{t}
$$

es la tasa promedio del indicador y $\mathrm{R}_{\mathrm{t}}$ es el rango relativo de $t_{\text {th }}$ nivel socioeconómico, definido como:

$$
R=\sum_{y=1}^{t-1} f_{r}+1 / 2 f_{t}
$$

e indica la proporción acumulada f de la población hasta el punto medio del intervalo de cada grupo.

\section{Resultados}

Las comparaciones hechas de la población colombiana revelaron diferencias importantes con respecto a los distintos indicadores de equidad analizados.

\section{Características de la población}

Las diferencias encontradas en la población colombiana con respecto a las variables demográficas, económicas, sociales y geográficas se presentan en Tabla 1. Con excepción del género, los dos cortes de la población tenidos en cuenta presentan diferencias en su estructura por edad, nivel de educación, deciles de ingreso y distribución geográfica. De modo similar, con la excepción del género, las comparaciones hechas entre los segmentos de la población afiliada a la seguridad social en salud muestran diferencias aún más acentuadas, con respecto a las demás características consideradas.

Desigualdades en salud

El análisis de indicadores de salud fue considerado dentro del presente estudio por tres razones fundamentales: (1) como medidas proxy de necesidad de atención en salud; (2) para evaluar sus efectos sobre la utilización de servicios; y, (3) para ser incluidos como variables de ajuste en el cálculo de los indicadores de prestación de servicios de salud. Los indicadores de salud considerados mostraron variaciones importantes en los dos sentidos de las comparaciones realizadas.

- Cambios observados entre 1993 y 1997. Observase un ligero aumento de los indicadores de salud entre 1993 y 1997 (Tabla 2). Por ejemplo, la prevalencia de morbilidad general ajustada (por edad, género y actividad restringida) aumentó de 10,7 a 12,9 entre 1993 y 1997, respectivamente; de modo similar, también se observó un aumento en la prevalencia de actividad restringida (por edad y género), de 4,7 a 5,7, durante el mismo período de tiempo.

Estas diferencias se mantienen en cada uno de los cuartiles de ingreso del hogar (Figura 1). Pero, adicionalmente, se observa un patrón importante asociado: tanto durante 1993 como 1997, la prevalencia ajustada de actividad restringida mostró un claro gradiente descendente en la medida que aumenta el ingreso; por esta razón, en el cálculo de los indicadores de equidad, además del género y de la edad, también se hizo un ajuste por morbilidad general o crónica con actividad restringida. En contraste con el patrón observado en la prevalencia de actividad restringida, Ilama la atención la poca variación observada en la prevalencia ajustada de morbilidad general. 
Tabla 1

Características demográficas y socio-económicas de la población afiliada a la seguridad social en salud. Colombia, 1993-1997.

\begin{tabular}{|c|c|c|c|c|c|c|c|c|c|c|c|c|}
\hline \multirow[t]{3}{*}{ Características } & \multicolumn{6}{|c|}{1993 (Casen) } & \multicolumn{6}{|c|}{1997 (Encalivida) } \\
\hline & \multicolumn{2}{|c|}{ Afiliados } & \multicolumn{2}{|c|}{ No afiliados } & \multicolumn{2}{|c|}{ Total } & \multicolumn{2}{|c|}{ Afiliados } & \multicolumn{2}{|c|}{ No afiliados } & \multicolumn{2}{|c|}{ Total } \\
\hline & $\mathrm{n}$ & $\%$ & $\mathrm{n}$ & $\%$ & $\mathrm{n}$ & $\%$ & $\mathrm{n}$ & $\%$ & $\mathrm{n}$ & $\%$ & $\mathrm{n}$ & $\%$ \\
\hline \multicolumn{13}{|l|}{ Género } \\
\hline Masculino & 4.253 .963 & 49,9 & 12.216 .829 & 49,0 & 16.470 .792 & 49,2 & 10.764 .172 & 47,2 & 8.553 .380 & 50,1 & 19.317 .552 & 48,5 \\
\hline Femenino & 4.264 .995 & 50,1 & 12.717 .728 & 51,0 & 16.982 .723 & 50,8 & 12.020 .046 & 52,8 & 8.504 .826 & 49,9 & 20.524 .872 & 51,5 \\
\hline Total & 8.518 .958 & 100,0 & 24.934 .557 & 100,0 & 33.453 .515 & 100,0 & 22.784 .218 & 100,0 & 17.058 .206 & 100,0 & 39.842 .424 & 100,0 \\
\hline \multicolumn{13}{|l|}{ Edad } \\
\hline$<1$ & 169.902 & 2,1 & 599.949 & 2,5 & 769.851 & 2,4 & 351.618 & 1,5 & 428.153 & 2,5 & 779.771 & 2,0 \\
\hline $1-4$ & 585.982 & 7,1 & 2.553 .366 & 10,5 & 3.139 .348 & 9,6 & 1.884 .974 & 8,3 & 1.611 .426 & 9,4 & 3.496 .400 & 8,8 \\
\hline $5-14$ & 1.584 .112 & 19,2 & 6.065 .020 & 24,9 & 7.649 .132 & 23,5 & 4.872 .996 & 21,4 & 3.934 .117 & 23,1 & 8.807 .113 & 22,1 \\
\hline $15-44$ & 4.397 .148 & 53,2 & 11.342 .525 & 46,7 & 15.739 .673 & 48,3 & 10.414 .036 & 45,7 & 8.198 .097 & 48,1 & 18.612 .133 & 46,7 \\
\hline $45-59$ & 1.114 .613 & 13,5 & 2.533 .161 & 10,4 & 3.647 .774 & 11,2 & 3.005 .622 & 13,2 & 1.646 .433 & 9,7 & 4.652 .055 & 11,7 \\
\hline $60 y+$ & 413.414 & 5,0 & 1.217 .229 & 5,0 & 1.630 .643 & 5,0 & 2.254 .972 & 9,9 & 1.239 .980 & 7,3 & 3.494 .952 & 8,8 \\
\hline Total & 8.265 .171 & 100,0 & 24.311 .250 & 100,0 & 32.576 .421 & 100,0 & 22.784 .218 & 100,0 & 17.058 .206 & 100,0 & 39.842 .424 & 100,0 \\
\hline \multicolumn{13}{|l|}{ Educación } \\
\hline Ninguna & 447.727 & 5,3 & 3.973 .451 & 15,9 & 4.421 .178 & 13,2 & 1.473 .022 & 6,5 & 1.630 .771 & 9,6 & 3.103 .793 & 7,8 \\
\hline Primaria & 2.453 .260 & 28,8 & 11.104 .911 & 44,5 & 13.558 .171 & 40,5 & 5.265 .630 & 23,1 & 4.851 .518 & 28,4 & 10.117 .148 & 25,4 \\
\hline Secundaria & 3.561 .579 & 41,8 & 6.021 .814 & 24,2 & 9.583 .393 & 28,6 & 5.622 .030 & 24,7 & 3.557 .827 & 20,9 & 9.179 .857 & 23,0 \\
\hline Universitaria & 1.014 .255 & 11,9 & 539.126 & 2,2 & 1.553 .381 & 4,6 & 1.234 .921 & 5,4 & 212.222 & 1,2 & 1.447 .143 & 3,6 \\
\hline Posgrado & 47.141 & 0,6 & 9.328 & 0,0 & 56.469 & 0,2 & 203.732 & 0,9 & 23.048 & 0,1 & 226.780 & 0,6 \\
\hline Sin información & 994.996 & 11,7 & 3.285 .927 & 13,2 & 4.280 .923 & 12,8 & 8.984 .883 & 39,4 & 6.782 .820 & 39,8 & 15.767 .703 & 39,6 \\
\hline Total & 8.518 .958 & 100,0 & 24.934 .557 & 100,0 & 33.453 .515 & 100,0 & 22.784 .218 & 100,0 & 17.058 .206 & 100,0 & 39.842 .424 & 100,0 \\
\hline \multicolumn{13}{|l|}{ Región } \\
\hline Atlántica & 1.733 .530 & 20 & 8.232 .290 & 33,0 & 9.965 .820 & 29,8 & 3.650 .597 & 16,0 & 4.871 .437 & 28,6 & 8.522 .034 & 21,4 \\
\hline O riental & 692.232 & 8,1 & 2.852 .274 & 11,4 & 3.544 .506 & 10,6 & 4.496 .179 & 19,7 & 2.394 .072 & 14,0 & 6.890 .251 & 17,3 \\
\hline Antioquia & 1.348 .501 & 15,8 & 3.185 .919 & 12,8 & 4.534 .420 & 13,6 & 4.038 .603 & 17,7 & 3.125 .880 & 18,3 & 7.164 .483 & 18,0 \\
\hline Central & 898.561 & 10,5 & 3.351 .321 & 13,4 & 4.249 .882 & 12,7 & 2.667 .174 & 11,7 & 2.476 .717 & 14,5 & 5.143 .891 & 12,9 \\
\hline Pacífica & 1.637 .000 & 19,2 & 4.232 .374 & 17,0 & 5.869 .374 & 17,5 & 3.417 .112 & 15,0 & 1.849 .953 & 10,8 & 5.267 .065 & 13,2 \\
\hline Bogotá & 2.144 .122 & 25,2 & 2.876 .374 & 11,5 & 5.020 .496 & 15,0 & 4.136 .812 & 18,2 & 2.187 .444 & 12,8 & 6.324 .256 & 15,9 \\
\hline O rinoquía & 45.489 & 0,5 & 181.054 & 0,7 & 226.543 & 0,7 & 310.212 & 1,4 & 134.283 & 0,8 & 444.495 & 1,1 \\
\hline San Andrés & 19.523 & 0,2 & 22.951 & 0,1 & 42.474 & 0,1 & 67.529 & 0,3 & 18.420 & 0,1 & 85.949 & 0,2 \\
\hline Total & 8.518 .958 & 100,0 & 24.934 .557 & 100,0 & 33.453 .515 & 100,0 & 22.784 .218 & 100,0 & 17.058 .206 & 100,0 & 39.842 .424 & 100,0 \\
\hline \multicolumn{13}{|l|}{ Deciles Ingreso } \\
\hline 10 & 91.803 & 1,2 & 2.406 .995 & 9,4 & 2.498 .798 & 7,5 & 1.328 .000 & 5,8 & 1.713 .324 & 10,0 & 3.041 .324 & 7,6 \\
\hline $2 \underline{0}$ & 111.849 & 1,4 & 2.904 .457 & 11,4 & 3.016 .306 & 9,0 & 1.453 .169 & 6,4 & 2.117 .550 & 12,4 & 3.570 .719 & 9,0 \\
\hline 30 & 377.317 & 4,8 & 2.431 .441 & 9,5 & 2.808 .758 & 8,4 & 1.760 .642 & 7,7 & 2.238 .426 & 13,1 & 3.999 .068 & 10,0 \\
\hline $4 \underline{0}$ & 480.376 & 6,1 & 3.027 .930 & 11,9 & 3.508 .306 & 10,5 & 1.898 .132 & 8,3 & 1.896 .022 & 11,1 & 3.794 .154 & 9,5 \\
\hline $5 \underline{0}$ & 592.172 & 7,5 & 2.783 .322 & 10,9 & 3.375 .494 & 10,1 & 2.019 .517 & 8,9 & 2.131 .014 & 12,5 & 4.150 .531 & 10,4 \\
\hline 60 & 717.850 & 9,0 & 2.200 .828 & 8,6 & 2.918 .678 & 8,7 & 2.428 .396 & 10,7 & 1.661 .024 & 9,7 & 4.089 .420 & 10,3 \\
\hline $7 \underline{0}$ & 1.063 .512 & 13,4 & 2.925 .314 & 11,5 & 3.988 .826 & 11,9 & 2.516 .728 & 11,0 & 1.733 .250 & 10,2 & 4.249 .978 & 10,7 \\
\hline 80 & 1.259 .682 & 15,9 & 2.428 .463 & 9,5 & 3.688 .145 & 11,0 & 2.806 .206 & 12,3 & 1.558 .698 & 9,1 & 4.364 .904 & 11,0 \\
\hline 90 & 1.506 .023 & 19,0 & 2.357 .433 & 9,2 & 3.863 .456 & 11,6 & 3.184 .762 & 14,0 & 1.161 .593 & 6,8 & 4.346 .355 & 10,9 \\
\hline 10 응 & 1.733 .560 & 21,8 & 2.047 .742 & 8,0 & 3.781 .302 & 11,3 & 3.388 .666 & 14,9 & 847.305 & 5,0 & 4.235 .971 & 10,6 \\
\hline Total & 7.934 .144 & 100,0 & 25.513 .925 & 100,0 & 33.448 .069 & 100,0 & 22.784 .218 & 100,0 & 17.058 .206 & 100,0 & 39.842 .424 & 100,0 \\
\hline \multicolumn{13}{|l|}{ Zona } \\
\hline Urbana & 7.793 .798 & 91,5 & 15.548 .036 & 62,4 & 23.341 .834 & 69,8 & 19.175 .292 & 84,2 & 13.330 .743 & 78,1 & 32.506 .035 & 81,6 \\
\hline Rural & 725.160 & 8,5 & 9.386 .521 & 37,6 & 10.111 .681 & 30,2 & 3.608 .926 & 15,8 & 3.727 .463 & 21,9 & 7.336 .389 & 18,4 \\
\hline Total & 8.518 .958 & 100,0 & 24.934 .557 & 100,0 & 33.453 .515 & 100,0 & 22.784 .218 & 100,0 & 17.058 .206 & 100,0 & 39.842 .424 & 100,0 \\
\hline
\end{tabular}

Casen = Encuesta Nacional de Caracterización Socio-económica; Encalivida = Encuesta de Calidad de Vida. 
Cambios observados en las tasas de salud y de utilización de servicios de salud (x 100), crudas y ajustadas, según afiliación a la seguridad social en salud y cuartiles de ingreso de los hogares en la población. Colombia, 1993-1997.

\begin{tabular}{|c|c|c|c|c|c|c|c|c|c|c|c|c|c|c|c|c|}
\hline \multirow[t]{3}{*}{ Indicadores } & \multicolumn{6}{|c|}{1993} & \multicolumn{10}{|c|}{1997} \\
\hline & \multicolumn{2}{|c|}{ Total } & \multicolumn{2}{|c|}{$\begin{array}{l}1993 \\
\mathrm{Si}\end{array}$} & \multicolumn{2}{|c|}{ No } & \multirow[b]{2}{*}{$\mathrm{RD}$} & \multirow[b]{2}{*}{$\begin{array}{l}\text { SE }(p< \\
0,05)\end{array}$} & \multicolumn{2}{|c|}{ Total } & \multicolumn{2}{|c|}{$\mathrm{Si}$} & \multicolumn{2}{|c|}{ No } & \multirow[b]{2}{*}{$\mathrm{RD}$} & \multirow[b]{2}{*}{ SE } \\
\hline & Tc & $\mathrm{Ta}$ & Tc & $\mathrm{Ta}$ & $\mathrm{Tc}$ & $\mathrm{Ta}$ & & & Tc & $\mathrm{Ta}$ & Tc & $\mathrm{Ta}$ & Tc & $\mathrm{Ta}$ & & \\
\hline \multicolumn{17}{|l|}{ Salud 1} \\
\hline \multicolumn{17}{|l|}{ Morbilidad } \\
\hline 10 & 16,3 & 11,3 & 22,5 & 15,5 & 15,9 & 11,1 & 1,5 & $*$ & 15,4 & 12,4 & 18,2 & 14,6 & 13,3 & 10,7 & 3,2 & * \\
\hline 20 & 15,4 & 10,8 & 19,8 & 13,9 & 14,6 & 10,3 & 1,9 & $*$ & 14,9 & 12,1 & 16,8 & 13,6 & 13,1 & 10,6 & 1,6 & * \\
\hline 30 & 15,2 & 10,6 & 19,6 & 13,7 & 13,7 & 9,5 & 1,5 & $*$ & 16,9 & 13,8 & 18,4 & 15,0 & 14,9 & 12,2 & 2,4 & * \\
\hline 40 & 14,5 & 9,9 & 18,1 & 12,3 & 12,5 & 8,6 & 1,5 & $*$ & 16,6 & 13,3 & 18,0 & 14,4 & 13,1 & 10,5 & 2,3 & $*$ \\
\hline Total & 15,4 & 10,7 & 19,2 & 13,2 & 14,4 & 10,0 & 1,4 & $*$ & 15,9 & 12,9 & 17,9 & 14,4 & 13,6 & 11,0 & 1,4 & * \\
\hline \multicolumn{17}{|c|}{ Actividad Restringida } \\
\hline 10 & 7,3 & 5,4 & 7,6 & 5,5 & 7,3 & 5,4 & 1,0 & $*$ & 10,7 & 7,0 & 11,9 & 7,9 & 9,3 & 6,3 & 1,3 & * \\
\hline $2 \underline{0}$ & 6,3 & 4,8 & 7,2 & 5,3 & 6,1 & 4,6 & 1,6 & $*$ & 9,1 & 6,2 & 10,1 & 6,9 & 8,0 & 5,6 & 1,6 & $*$ \\
\hline 30 & 6,1 & 4,7 & 6,9 & 5,0 & 5,9 & 4,4 & 1,2 & $*$ & 9,3 & 6,6 & 10,0 & 6,9 & 8,8 & 6,2 & 1,1 & $*$ \\
\hline $4 \underline{0}$ & 5,6 & 4,3 & 6,4 & 4,5 & 5,3 & 3,9 & 1,2 & $*$ & 7,5 & 5,7 & 9,1 & 6,0 & 7,3 & 5,0 & 1,2 & * \\
\hline Total & 6,4 & 4,7 & 6,8 & 4,9 & 6,2 & 4,6 & 1,1 & $*$ & 9,4 & 6,4 & 10,1 & 6,8 & 8,5 & 5,9 & 1,2 & * \\
\hline \multicolumn{17}{|c|}{$\begin{array}{l}\text { Estado general de } \\
\text { salud regular o malo }\end{array}$} \\
\hline 10 & - & - & - & - & - & - & & & 45,1 & 19,7 & 47,0 & 20,3 & 42,9 & 19,2 & 3,2 & * \\
\hline 20 & - & - & - & - & - & - & & & 36,7 & 15,8 & 37,0 & 15,4 & 36,3 & 16,1 & 1,6 & * \\
\hline 30 & - & - & - & - & - & - & & & 29,5 & 11,3 & 28,6 & 10,5 & 30,1 & 12,4 & 2,4 & * \\
\hline 40 & - & - & - & - & - & - & & & 21,3 & 6,2 & 19,1 & 5,5 & 22,2 & 7,9 & 2,3 & * \\
\hline Total & - & - & - & - & - & - & & & 32,6 & 16,1 & 30,9 & 20,2 & 34,7 & 10,9 & 2,1 & * \\
\hline \multicolumn{17}{|c|}{$\begin{array}{l}\text { Utilización servicios } \\
\text { de salud2 }\end{array}$} \\
\hline \multicolumn{17}{|c|}{ Hospitalización } \\
\hline 10 & 3,7 & 1,5 & 6,1 & 2,5 & 3,5 & 1,5 & 1,7 & $*$ & 6,5 & 3,0 & 3,0 & 3,4 & 5,6 & 2,7 & 1,2 & $*$ \\
\hline 20 & 3,8 & 1,6 & 5,8 & 2,5 & 3,4 & 1,5 & 1,6 & $*$ & 6,8 & 3,3 & 3,3 & 3,9 & 5,5 & 2,8 & 1,4 & * \\
\hline 30 & 4,0 & 1,7 & 6,1 & 2,6 & 3,3 & 1,4 & 1,8 & $*$ & 7,0 & 3,4 & 3,4 & 4,2 & 4,7 & 2,4 & 1,8 & $*$ \\
\hline 40 & 4,3 & 1,8 & 5,6 & 2,3 & 3,5 & 1,6 & 1,5 & $*$ & 7,8 & 3,8 & 3,8 & 4,2 & 5,3 & 2,7 & 1,6 & $*$ \\
\hline Total & 3,9 & 1,7 & 5,8 & 2,5 & 3,4 & 1,5 & 1,7 & $*$ & 7,0 & 3,4 & 8,4 & 4,0 & 5,3 & 2,7 & 1,5 & * \\
\hline \multicolumn{17}{|c|}{ Servicios de Salud } \\
\hline 10 & 12,0 & 5,3 & 23,3 & 13,9 & 11,3 & 4,8 & 3,2 & * & 17,9 & 7,4 & 22,1 & 9,2 & 14,8 & 6,0 & 1,6 & * \\
\hline $2 \underline{0}$ & 13,8 & 7,2 & 22,6 & 13,7 & 12,1 & 5,9 & 1,6 & $*$ & 20,9 & 10,1 & 26,4 & 13,4 & 15,6 & 7,1 & 1,0 & $*$ \\
\hline 30 & 15,1 & 8,4 & 22,7 & 14,0 & 12,5 & 6,4 & 2,4 & * & 25,0 & 12,8 & 30,0 & 16,1 & 18,1 & 8,4 & 2,1 & * \\
\hline $4 \underline{0}$ & 16,8 & 9,9 & 23,6 & 15,0 & 13,0 & 7,1 & 2,3 & $*$ & 33,0 & 18,8 & 37,6 & 22,3 & 21,4 & 11,1 & 2,2 & * \\
\hline Total & 14,4 & 7,7 & 23,1 & 14,4 & 12,1 & 5,9 & 2,7 & $*$ & 24,2 & 12,4 & 30,1 & 16,1 & 16,9 & 7,8 & 2,3 & $*$ \\
\hline
\end{tabular}

$\mathrm{TC}=$ Tasa cruda; $\mathrm{Ta}$ = Tasa ajustada; RD = Razón de disparidad; $\mathrm{SE}$ = Significancia estadística.

1 Tasas ajustadas para edad y género por regresión logística.

2 Tasas ajustadas para edad, género y actividad restringida por regresión logística.

$*(p<0,05)$ 
Figura 1

Cambios observados en las tasas de utilización de servicios y de actividad restringida según cuartiles de ingresos del hogar. Colombia, 1993-19971.

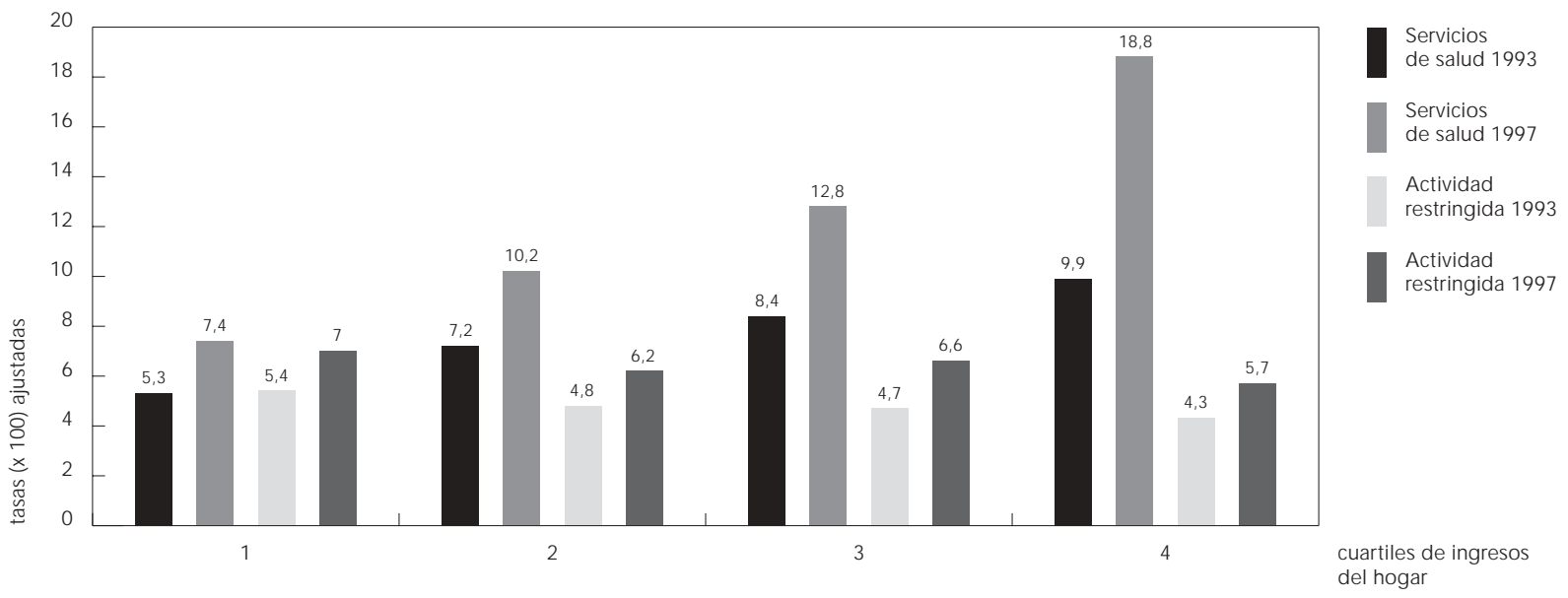

1 Tasas (X 100) ajustadas por regresión logística; las tasas de utilización están ajustadas por edad, género y actividad restringida; las tasas de actividad restringida están ajustadas por edad y género.

Diferencias entreafiliadosy no afiliadosa la seguridad social. Se observa un aumento en los indicadores de salud, con respecto a los que no están afiliados, en ambos cortes de tiempo considerados (Tabla 2). Durante 1993, por ejemplo, las tasas ajustadas de prevalencia de morbilidad general y de actividad restringida fueron, respectivamente, 1,4 y 1,1 veces más altas entre los afiliados a la seguridad social, en comparación con los que no lo están; de forma similar, durante 1997, las tasas ajustadas de prevalencia de morbilidad general y de actividad restringida fueron, respectivamente, 1,4 y 1,1 veces más altas entre los afiliados a la seguridad social, en comparación con los que no lo están. En ambos casos, las RD correspondientes fueron estadísticamente significativas ( $p<0,05)$.

Adicionalmente, en cada uno de los dos períodos de tiempo considerados, las diferencias descritas en las prevalencias de morbilidad y de actividad restringida se mantienen en cada uno de los cuatro deciles de ingreso considerado. Sin embargo, hay diferencias importantes en los patrones observados. Durante 1993, por ejemplo, la prevalencia ajustada de morbilidad general mostró una fuerte asociación con el nivel de ingresos de la población entre el grupo afiliado a la seguridad social: la prevalencia varió de 22,5 en el primer decil de ingresos de los hogares y cayó progresivamente a 18,1 en el último cuartil de más altos ingresos; este patrón contrasta con el observado en el grupo que no está afiliado a la seguridad social, en donde hubo muy poca variación: la prevalencia de morbilidad varió en forma progresiva de 11,1 a 8,6, entre el primero y el último cuartil de ingresos, respectivamente.

Durante 1997, también se observaron niveles consistentemente superiores en la prevalencia ajustada de morbilidad entre el grupo de personas afiliadas a la seguridad social con respecto a las que no lo están; sin embargo, las diferencias encontradas en ambos grupos fueron mínimas en relación con el nivel de ingresos.

En forma similar al caso anterior, también se observó una asociación positiva en la prevalencia ajustada de actividad restringida, con respecto al estado de afiliación a la seguridad social y al nivel de ingresos de los hogares.

\section{Equidad en el acceso a los servicios}

Para evaluar los resultados de la reforma sobre la equidad en el acceso a los servicios de salud, se evaluó la distribución de dos indicadores proxy: (1) estar afiliado o no a una empresa aseguradora del SGSSS, y (2) las razones para no utilizar los servicios de salud en caso necesidad.

La cobertura en la afiliación pasó del 23,7\% durante 1993 al 57,2\%, en 1997 (Tabla 3). Este incremento observado en la cobertura del SGSSS afectó a las personas en todos los deci- 
Cambios en la cobertura de la seguridad social en salud según deciles de ingreso del hogar, Colombia, 1993-1997.

\begin{tabular}{|c|c|c|c|c|c|c|c|c|}
\hline \multirow[t]{2}{*}{ Deciles } & \multicolumn{4}{|c|}{1993} & \multicolumn{4}{|c|}{1997} \\
\hline & $\mathrm{n}$ & $\%$ & RD & SE & $\mathrm{n}$ & $\%$ & RD & SE \\
\hline 1 & 3.801 .716 & 3,1 & 1,0 & $*$ & 3.041 .324 & 43,7 & 1,0 & $*$ \\
\hline 2 & 2.956 .170 & 9,2 & 3,2 & $*$ & 3.570 .719 & 40,7 & 0,6 & $*$ \\
\hline 3 & 3.257 .018 & 12,6 & 4,6 & $*$ & 3.999 .068 & 44,0 & 0,5 & $*$ \\
\hline 4 & 3.368 .185 & 14,5 & 5,3 & $*$ & 3.794 .154 & 50,0 & 0,6 & $*$ \\
\hline 5 & 3.583 .381 & 22,8 & 9,3 & $*$ & 4.150 .531 & 48,7 & 0,8 & $*$ \\
\hline 6 & 3.110 .062 & 25,5 & 10,1 & $*$ & 4.089 .420 & 59,4 & 0,7 & $*$ \\
\hline 7 & 3.373 .438 & 29,3 & 13,0 & $*$ & 4.249 .978 & 59,2 & 1,1 & $*$ \\
\hline 8 & 3.323 .844 & 33,9 & 16,2 & $*$ & 4.364 .904 & 64,3 & 1,1 & * \\
\hline 9 & 3.332 .152 & 42,1 & 23,0 & $*$ & 4.346 .355 & 73,3 & 1,4 & * \\
\hline 10 & 3.347 .549 & 45,4 & 26,0 & $*$ & 4.235 .971 & 80,0 & 2,0 & * \\
\hline Total & 33.453 .515 & 23,7 & & & 39.842 .424 & 57,2 & & \\
\hline
\end{tabular}

$\mathrm{RD}=$ Razón de disparidad u odds ratios que está asociada con el decil de ingreso; $\mathrm{SE}$ = significancia estadística. $*(p<0,05)$

les de ingreso del hogar; pero fue mucho más dramático en el primer decil de ingresos, en donde la cobertura pasó del 3,1\% al 43,7\%, entre 1993 y 1997, respectivamente. Este comportamiento se expresa como un gradiente positivo en el comportamiento de la cobertura, asociado con el decil de ingresos de los hogares (es decir, la cobertura aumenta en relación con el ingreso); sin embargo, el gradiente fue mucho menos pronunciado durante 1997, en comparación con 1993. También se expresa como un aplanamiento de la curva de distribución de la afiliación al SGSSS con respecto a la diagonal y, a la vez, como un descenso en el índice de concentración correspondiente, el cual pasó de 0,34, en 1993, a 0,17 en 1997 (Figura 2).

Las razones para no utilizar los servicios de salud proporcionan una visión complementaria y miden la presencia de barreras económicas, funcionales y geográficas que dificultan la accesibilidad a los servicios de salud. Al comparar los segmentos de la población afiliada y no afiliada a la seguridad social en salud durante 1997, con respecto a las tasas de no uso ajustadas por edad, género y actividad restringida, se observa que, entre los segmentos no cubiertos por la seguridad social en salud, la falta de dinero constituye la principal razón para no utilizar los servicios de salud, en tanto que, entre los afiliados, es la segunda razón más importante.

\section{Equidad en la utilización de servicios}

Los resultados de la reforma sobre la equidad en la accesibilidad y la utilización final de los servicios de salud fueron evaluados a través de tres indicadores: (1) utilización de servicios hospitalarios en el último año; (2) utilización de servicios de salud ambulatorios en los últimos 30 días; y (3) también, razones para no utilizar los servicios de salud.

Ambos indicadores de utilización mostraron incrementos importantes entre los dos cortes de tiempo considerados. Entre 1993 y 1997, las tasas ajustadas (x 100) de hospitalización y de servicios de salud pasaron de 1,7 a 3,4 y de 7,7 a 12,4 , respectivamente (Tabla 2). De otro lado, también se observaron incrementos en la utilización asociados con la afiliación a la seguridad social. Por ejemplo, durante 1997, las razones de disparidad correspondientes a la utilización de servicios de hospitalización y de salud, asociadas con la afiliación a la seguridad social en salud, fueron $1,5(p<0,05)$ y 2,3 ( $p<$ 0,05 ), respectivamente; estas magnitudes en las razones de disparidad registradas durante 1997 fueron inferiores a las observadas durante 1993.

Adicionalmente a la afiliación a la seguridad social en salud, la utilización de servicios está fuertemente asociada con las variables proxy de necesidades de salud: (1) morbilidad general; (2) morbilidad general ó crónica con actividad restringida; $y$, (3) estado general de salud regular o malo. En este sentido, las tasas ajustadas de hospitalización y de uso de servicios de salud muestran incrementos sustanciales, de por lo menos dos órdenes de magnitud, estadísticamente significativos, cuando los indicadores de salud son positivos. Por ejemplo, durante 1997, las tasas ajustadas correspondientes a uso de servicios de salud fueron: $70,2 \%$ 
Figura 2

Curvas de concentración correspondientes a la distribución de la afiliación a la seguridad social en salud por deciles de ingresos del hogar. Colombia, 1993-19971.

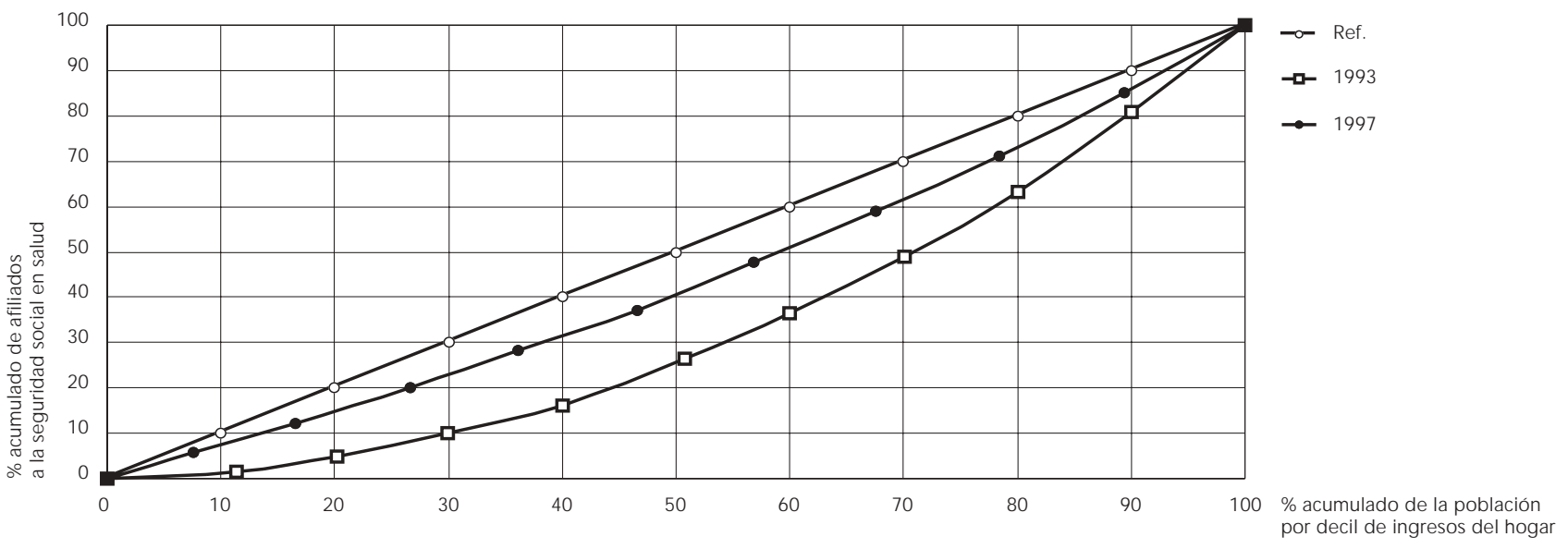

1 Índices de concentración para 1993 y 1997: 0,34 y 0,17, respectivamente.

y $12,4 \%$, entre las personas con y sin actividad restringida, respectivamente.

La distribución observada en los indicadores de utilización de servicios de salud revela una fuerte asociación positiva con el decil de ingresos del hogar (Figura 1). Esta asociación disminuye, pero persiste, luego del ajuste hecho por diferencias de edad, género y actividad restringida. También se expresa como cambios (durante el período de tiempo considerado), en las curvas de distribución de las curvas de utilización (con respecto a la diagonal), y en losíndices de concentración correspondientes. Por ejemplo, entre 1993 y 1997, se observa una ligera modificación en la curva correspondiente a la distribución de la utilización de servicios hospitalarios, con respecto a la distribución de la población según deciles de ingresos del hogar, tendencia que se manifiesta como un descenso en el índice de concentración, de-0,028 a -0,013 (Figura 3); en contraste, la curva correspondiente a la distribución de la utilización de servicios de salud en general muestra un cambio mayor, pero en el sentido opuesto: el índice de concentración aumentó de -0,038 a 0,091 (Figura 4).

De otro lado, los incrementos observados en las tasas de utilización de servicios son mayores en el caso de los servicios de salud ambulatorios con respecto a los servicios de hospitalización. Estas diferencias son aún más acentuadas entre la población afiliada a la seguri- dad social en salud, con respecto a la que no lo está, y durante 1997 con respecto a 1993.

\section{Discusión}

Las evidencias aportadas por la presente investigación sugieren tres conclusiones. Primero, la reforma de salud colombiana tuvo un impacto importante sobre el acceso a los servicios de salud, como resultado del incremento importante observado en la cobertura del aseguramiento a partir de 1993, especialmente entre los segmentos más pobres y vulnerables de la población. Segundo, no se evidenció en forma clara un impacto sobre la equidad en la accesibilidad y en la utilización final de servicios. Tercero, el aseguramiento está generando un consumo excesivo de servicios de salud, superior al óptimo social desde el punto de vista económico, el cual se explica por la presencia de dos fenómenos que plagan el aseguramiento en todo el mundo, a saber: selección adversa y riesgo moral.

Equidad en el acceso a través del aseguramiento

El impacto sobre el acceso a la red de instituciones prestadoras de servicios de salud se reflejó en el comportamiento de la cobertura del aseguramiento: el índice de concentración cor- 
Figura 3

Curvas de concentración correspondientes a la distribución de servicios hospitalarios por deciles de ingresos del hogar. Colombia, 1993-19971.

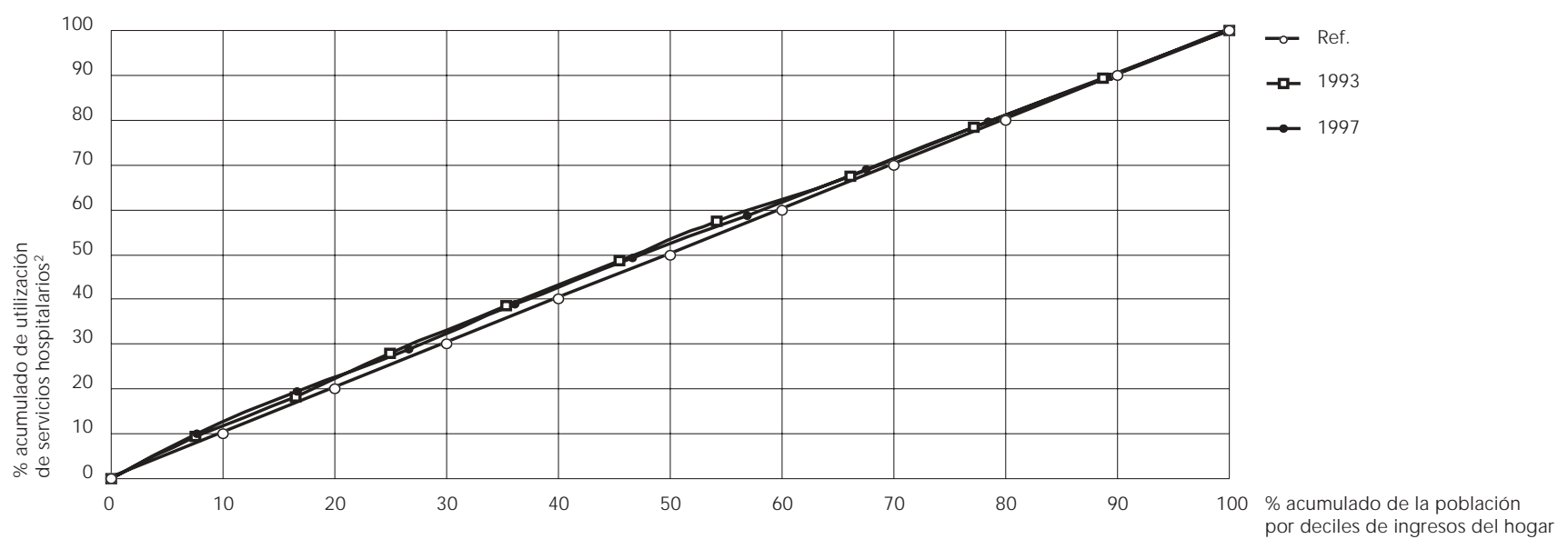

1 Índices de concentración para 1993 y 1997: -0,028 y -0,013, respectivamente.

Figura 4

Curvas de concentración correspondientes a la distribución de la utilización de servicios de salud ambulatorios por deciles de ingresos del hogar. Colombia, 1993-19971.

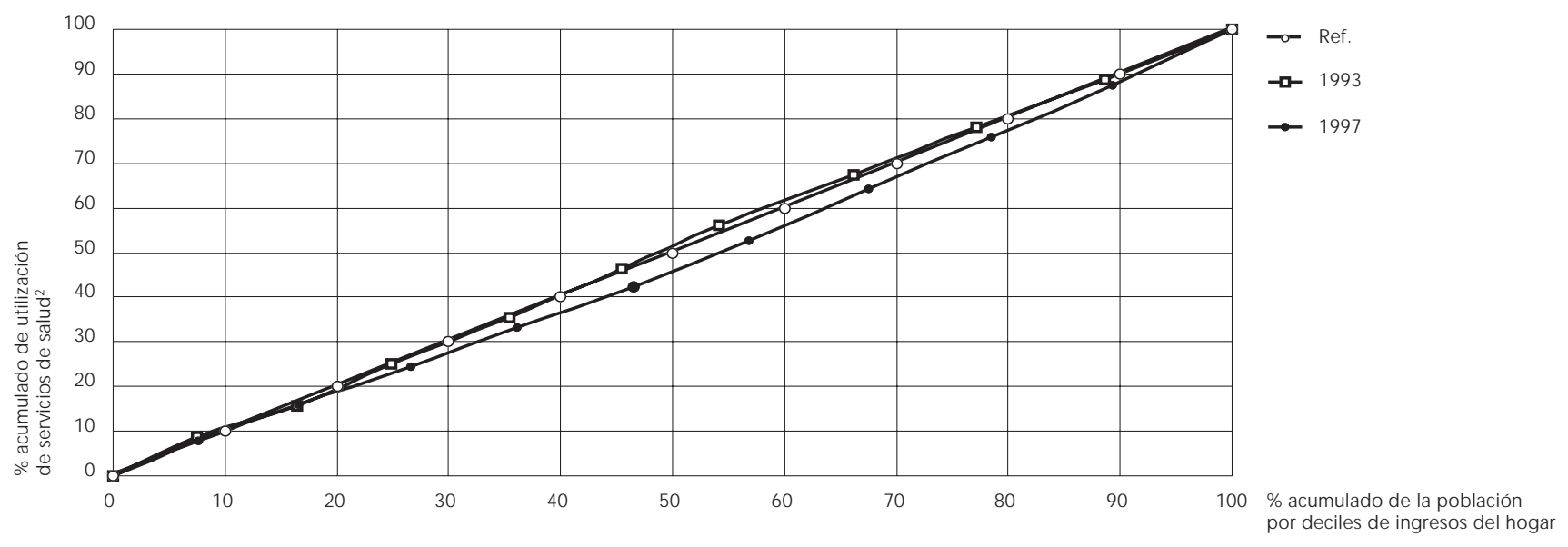

1 Índices de concentración para 1993 y 1997: -0,056 y 0,091, respectivamente. 
respondiente a la distribución de afiliados al SGSSS se redujo a la mitad, de 0,34 a 0,17. Este impacto se explica porque, entre 1993 y 1997, la cobertura del aseguramiento pasó del 20 al 57 por ciento de la población. Es más, el incremento observado en la cobertura fue mucho mayor entre las personas que viven en los hogares con menores ingresos. Este último hecho, a su vez, refleja la eficacia de las políticas distributivas puestas en marcha por el Gobierno a partir de 1993, con el propósito de eliminar las barreras económicas que dificultaban el acceso a los servicios de salud entre los segmentos dela población sin capacidad de pago suficiente; por ejemplo, durante 1997, el SGSSS Iogró otorgar casi 7,0 millones de subsidios, previamente focalizados a los individuos más pobres y vulnerables de la población, utilizando para ello el SISBEN (Reyes et al., 1998).

No obstante, aún queda un largo camino por recorrer frente a los objetivos y metas trazados por la reforma. Particularmente, la meta de cobertura universal, fijada inicialmente para 2000, está aún lejana: cerca del $40 \%$ de la población está por fuera del SGSSS, principalmente como resultado de no poseer un vínculo laboral formal (Nieto-López \& Londoño, 2000).

Adicionalmente, la persistencia de la falta de dinero como causa importante para no utilizar los servicios de salud, cuando las personas los necesitan (inclusive entre la población asegurada), plantea dudas sobre los posibles efectos que puedan estar teniendo las cuotas moderadoras y copagos. Estas herramientas hacen parte de los mecanismos de atención gerenciada orientados a la demanda puestos en marcha para controlar los costos derivados del efecto de riesgo moral (que serán analizados más adelante), pero podrían estar restringiendo el acceso a los servicios más allá de lo deseado.

Equidad en la utilización final de servicios

Entre 1993 y 1997, el índice de concentración correspondiente a la distribución de servicios hospitalarios bajó de-0,028 a -0,013, lo cual indicaría un efecto positivo sobre la equidad. En cambio, el índice de concentración correspondiente a la distribución de la utilización de servicios de salud ambulatorios aumentó de 0,038 a 0,091, durante el mismo período de tiempo, lo cual indicaría un efecto negativo.

Estos valores, tan cercanos a cero, observados en los índices de concentración, se expresan gráficamente como curvas de concentración muy cercanas a la línea recta diagonal que representa una distribución equitativa. Sin em- bargo (y en contraste con los índices de concentración en el aseguramiento, donde las diferencias observadas fueron sustanciales, el comportamiento poco expresivo observado en los indicadores de utilización de servicios), no se puede interpretar claramente como la consecuencia de un impacto positivo del nuevo SGSSS sobre la utilización de servicios, por dos razones fundamentales.

Primero, los bajos índices de concentración observados en la utilización de servicios de salud podrían significar que, en 1993 (cuando apenas se iniciaba la implementación del SGSSS correspondiente a la segunda etapa de la reforma), ya se observaban altos niveles de equidad en la utilización de servicios en Colombia, y que su variación subsiguiente ha sido muy poca. Esto seria contradictorio con la propia justificativa de la reforma. Pero, cabe la posibilidad de que si puedan ser atribuidos a los esfuerzos de descentralización y modernización de la red pública de instituciones prestadoras de servicios, iniciados durante la primera etapa de la Reforma comenzada a partir de 1990 (Bossert et al., 2000; Sánchez \& Mendez, 1999); en efecto, con anterioridad a 1990, el sistema de salud colombiano se caracterizaba por su ineficiencia, mala calidad e ineficiencia (Yepes, 1999).

Esta interpretación es coherente con otros estudios realizados en Colombia, utilizando indicadores diferentes, tales como la concentración del gasto social (Molina et al., 1993; Sarmiento, 1999; Vélez, 1994) y del gasto en salud, particularmente (Castaño et al., 1999), aunque otros estudios no están de acuerdo (Málaga et al., 2000). También es coherente con los resultados esperados de las políticas macroeconómicas y sectoriales que fueron adoptadas en Colombia durante la pasada década y enmarcaron la reforma. En particular, cabe resaltar el hecho de que la no afiliación al SGSSS no supone una barrera para acceder al sistema de prestación de servicios de salud colombiano. Por el contrario, las personas que no están afiliadas al régimen contributivo $\mathrm{ni}$ al subsidiado pueden acceder a la red pública de instituciones prestadoras de servicios de otra forma, denominada régimen de "vinculados" o seguridad social ampliada (Londoño \& Frenk, 1997). Dicho régimen viene experimentando, desde 1990, un proceso importante de descentralización y modernización institucional, como se mencionó.

Segundo, pese a lo expuesto, no se pueden descartar otras interpretaciones. Por ejemplo, los resultados podrían explicarse por error de muestreo, por tratarse de una encuesta multipropósito y no de una encuesta de salud (tanto 
en 1993 como en 1997). Por tanto, la proporción de encuestados que tenían problemas de salud y sus sucesivas desagregaciones podrían Ilevar a encontrar resultados inesperados; en este sentido, es importante recalcar la necesidad de una encuesta periódica de salud que permita hacerle seguimiento a importantes indicadores de utilización de servicios de salud. Finalmente, también cabe la posibilidad de que los efectos del SGSSS sobre la utilización se expresen con mayor claridad a través de otros indicadores, que no fueron abordados por la presente investigación, y permitan capturar mayores detalles sobre la calidad técnica: oportunidad e integralidad de la atención, y gasto en salud.

Consumo excesivo de servicios debido a la selección sesgada de riesgos y el riesgo moral

De otro lado, el presente estudio también reveló la presencia de fenómenos propios de los seguros privados que afectan el comportamiento del SGSSS colombiano: selección sesgada de riesgos y riesgo moral.

Los fenómenos de selección sesgada de riesgos plagan los sistemas de aseguramiento durante la transición hacia la meta de cobertura universal (Castaño, 1999; Feldstein, 1998; Hsiao, 1994). Adoptan una de dos formas: selección adversa por parte de los asegurados y selección de riesgos (o descreme) por parte de los aseguradores.

La selección adversa se debe a las asimetrías de información por parte de los consumidores con respecto a los aseguradores, debido a que los primeros usualmente conocen mejor sus propios riesgos de enfermar que los segundos. Por tanto, el grado de certidumbre frente al riesgo de enfermar se convierte en un factor determinante a la hora de comprar o afiliarse a un sistema de aseguramiento, lo cual resulta análogo a adquirir un seguro una vez el vehículo ha sido chocado. La selección adversa expone a las empresas aseguradoras a una mayor concentración de la siniestralidad con respecto a la población general, lo cual invalida las estimaciones actuariales y financieras. En Colombia, por ejemplo, la población afiliada al SGSSS, comparada con el resto, experimenta un riesgo de enfermar entre 1,2 y 2,1 veces mayor.

Adicionalmente, la selección de riesgos (o descreme) es utilizada por las aseguradoras como forma de minimizar sus costos, y lo cual genera distorsiones en la distribución de la siniestralidad al interior del SGSSS. Por ejemplo, al menos un $63 \%$ de los eventos por enfermedades de alto costo se concentra en el Instituto de Seguros Sociales (ISS), la EPS más grande de Colombia, con cerca 4,5 millones de afiliados (Céspedes, 2001). Esto se explica porque muchas EPS en Colombia, para protegerse de la selección adversa, niegan la afiliación o la renovación de la póliza a personas con condiciones preexistentes, con lo cual segmentan el mercado e inducen a un movimiento de personas con alto riesgo de siniestralidad hacia las EPS públicas, especialmente el ISS. La mayoría de las prácticas de descreme están prohibidas en el SGSSS; sin embargo, las agencias estatales responsables de inspección, vigilancia y control no son suficientemente eficaces para evitarlo.

El riesgo moral, que genera la utilización excesiva de servicios de salud, es el otro fenómeno que resultó claramente evidenciado en la presente investigación. Esto se explica porque la presencia de un tercero (el asegurador), que paga la factura al prestador de servicios, disminuye el costo directo para el asegurado, es decir, su gasto de bolsillo y el precio que paga para acceder a los servicios de salud, lo cual se traduce en un aumento en la cantidad demandada de servicios (Fuchs, 1998). En CoIombia, por ejemplo, la tasa ajustada de utilización de servicios observada entre la población afiliada al SGSSS es 2,7 veces mayor con respecto a la que no lo está. Este último hecho conlleva a un desperdicio de los escasos recursos del SGSSS que atenta contra el principio de eficiencia en su asignación, especialmente los recursos disponibles para subsidiar la afiliación de las personas más pobres y vulnerables de la población.

\section{Implicaciones para la política}

De la exposición y del análisis anteriores resulta evidente que hay avances importantes logrados por la reforma de salud colombiana. Esto contrasta con los escasos logros obtenidos por el antiguo sistema nacional de salud (que algunos defienden todavía) en las dos décadas previas (Yepes, 2000). También es aparente la imperiosa necesidad de introducir ajustes al proceso, con el fin de completar la agenda política prevista.

Concretamente, la situación expuesta plantea la siguiente reflexión a los investigadores, planificadores y formuladores de políticas de salud en relación con las opciones disponibles para mejorar los niveles de equidad en el acceso al SGSSS: (1) ¿Qué tipo de ajustes se requieren? (2) ¿A qué nivel se deben dar dichos ajustes: diseño del SGSSS (es decir, revisar y ajustar el marco legal), o implementación (es decir, 
aplicar con mayor eficacia los instrumentos existentes)?

En tal sentido, aunque el nuevo SGSSS enfrenta enormes retos (que han sido señalados por muchos autores), estos no parecen originarse del diseño del Sistema: ausencia de políticas e instrumentos que respondan en forma pertinente a las necesidades de atención de la población. Dicho de otro modo, el problema fundamental no radica en términos de diseño del Sistema, aunque si se requieren algunos ajustes. Más que reformas adicionales ó, inclusive, una contrarreforma (como algunos han Ilegado a plantear), lo que se requiere fundamentalmente es redoblar los esfuerzos dirigidos a mejorar la aplicación efectiva de los instrumentos y reglas de juego vigentes.

Por consiguiente, los ajustes requeridos (con el fin de reducir, aún más, las inequidades que persisten en el acceso a los servicios de salud) deben ir encaminados principalmente a consolidar el proceso de reforma (Céspedes, 1998b). Las acciones necesarias se deben enfocar fundamentalmente a mejorar la aplicación y la implementación de los instrumentos y de las reglas de juego existentes que enmarcan la operación de los regímenes subsidiado y contributivo del SGSSS, como el régimen de vinculados.

El régimen subsidiado es el más crítico para el logro de los objetivos de equidad. El sistema dispone de recursos suficientes y, además, cuenta con las herramientas necesarias para focalizarlos (SISBEN) hacia los segmentos más pobres y vulnerables de la población (Alvarado, 1998; Jaramillo, 1999). Sin embargo, la aplicación de estos recursos se hace con altos niveles de ineficiencia: en los años recientes, sólo el $50 \%$ de los recursos destinados al régimen subsidiado se ha traducido en servicios de salud que beneficien a la población más pobre y vulnerable. La ineficiencia se explica principalmente por la larga cadena de operaciones administrativas (originada en las agencias nacionales y terminada en las subnacionales), que ocasiona fugas en los recursos. Para atacar el problema, se precisan algunos ajustes en términos de diseño, con el fin de introducir procesos administrativos menos complejos; sin embargo, donde hay que actuar principalmente es en Ias Direcciones Territoriales de Salud, con fin de fortalecer sus procesos de supervisión y control. En esta dirección, con la consolidación del proceso de descentralización en Colombia y el fortalecimiento operativo de las agencias reguladoras subnacionales, cabe esperar una mayor eficiencia del gasto social destinado a subsidiar el aseguramiento de los segmentos más pobres de la población.
En el régimen contributivo, el reto fundamental para aumentar la cobertura consiste en cómo hacer cumplir las regulaciones previstas, con el fin de enfrentar el obstáculo principal: los altos niveles de evasión y elusión de los aportes obligatorios al SGSSS (de hecho, superiores a un $30 \%$, en comparación con el recaudo esperado), principalmente por parte de los trabajadores con capacidad de pago suficiente e independientes (Reyes \& Cespedes, 1999). Esta situación compromete, adicionalmente, los recursos de solidaridad que los afiliados a ese régimen deben aportar obligatoriamente al SGSSS. En términos de diseño, se requiere simplificar el proceso de recaudo de aportes al SGSSS y de compensación de la UPC con el FOSYGA, a través de un proceso único, menos complejo y mejor soportado en tecnología de informática.

No obstante, el problema fundamental a atacar, en ambos regímenes, es la falta de legitimidad, imparcialidad, autoridad, autonomía y capacidad, por parte de las agencias estatales especializadas - como la SNS y las Direcciones Territoriales de Salud (DTS) - para supervisar y hacer cumplir las reglas de juego existentes (Bitrán \& Céspedes, 2001). Esta situación dificulta que las instituciones y los diferentes actores que conforman el SGSSS se comporten y funcionen en la dirección que la mayoría de los miembros de la sociedad lo desean, y han expresado explícitamente a través de sus leyes y normas. A estos factores, que diezman la capacidad para supervisar y controlar el cumplimiento de las regulaciones y reglas de juego del Sistema, se suma otro: la captura de dichas agencias por intereses políticos y privados. La respuesta a este último problema representa uno de los principales retos de política pública que encara la reforma del SGSSS en Colombia (Harvard University, 1996); de lo contrario, el cuerpo de normas y regulaciones vigentes que definen las reglas de juego del SGSSS se convertirá en letra muerta.

De otro lado, el fortalecimiento de la operación del régimen de los vinculados (o seguridad social ampliada) resulta igualmente crítico para garantizar el acceso de la población a los servicios de salud. Cabe la posibilidad de que, en cerca de la mitad del territorio nacional, donde viven al menos el $10 \%$ de los colombianos (en condiciones de dispersión y marginamiento), el aseguramiento no funcione adecuadamente; sin embargo, la red pública prestadora de servicios de salud Ilega hasta estos lugares más remotos de la geografía colombiana. Es más, como resultado del proceso de descentralización iniciado en 1990, la mayoría de las IPS públicas de mayor complejidad se han transfor- 
mado en Empresas Sociales del Estado (ESE), con autonomía jurídica, financiera y administrativa, para competir con las demás IPS y atender las necesidades de salud de la población con eficiencia y calidad (Britán \& Céspedes, 2001); sin embargo, por lo menos la mitad de las IPS públicas de menor complejidad no lo ha hecho todavía, debido a la pobre capacidad de la mayoría de los gobiernos locales, sumada al poco apoyo que han recibido de los gobiernos territoriales y nacional. Para avanzar en esta última dirección, se requiere: (1) ajustar la ley 60 de 1993, con el fin de modificar el flujo de recursos financieros, de tal manera que los recursos sean asignados en función de la población atendida y, además, Ileguen directamente a los Fondos Locales de Salud, y, (2) fortalecer la capacidad operativa de las Direcciones Locales de Salud, para supervisar y controlar el comportamiento de sus sistemas de salud y seguridad social (Britán \& Céspedes, 2001).

Las políticas planteadas arriba también tendrían efectos positivos sobre el consumo excesivo de servicios, originado en los fenómenos de selección sesgada de riesgos y riesgo moral. En respuesta a este problema, el SGSSS previó todo un arsenal de herramientas de atención gerenciada (managed care), cuya efectividad ha sido ampliamente demostrada en otras partes del mundo (Daniels, 1996; DeMuro, 1995). De hecho, las EPS más exitosas en Colombia vienen aplicando muchas de estas herramientas (Britán \& Céspedes, 2001). Sin embargo, se re- quieren mayores esfuerzos enfocados a fortalecer la capacidad de las agencias estatales responsables de supervisar y controlar su aplicación y lograr que tanto aseguradores como prestadores de servicios de salud ajusten sus procesos en esta dirección.

En suma, las evidencias analizadas sugieren una mejora sustantiva en la equidad en los servicios de salud en Colombia, como resultado de la reforma en salud. Para consolidar los avances logrados, es preciso redoblar los esfuerzos en relación con los dos aspectos de la reforma que más han contribuido a ello: el proceso de descentralización y la regulación del funcionamiento del nuevo SGSSS. De otro lado, es necesario refinar las metodologías de evaluación disponibles, lo cual implica estudios adicionales empleando otros indicadores de utilización de servicios, que no fueron abordados por la presente investigación, tales como: oportunidad e integralidad de la atención, y gasto en salud (Wagstaff \& van Doorslaer, 1998; Wagstaff et al., 1991a, 1991b, 1992, 1999). Adicionalmente, es importante considerar en futuras investigaciones otras dimensiones de la equidad, como las diferencias en la utilización de servicios con respecto a género, edad, educación y regiones del país. Finalmente, también es necesario investigar el posible efecto de los mecanismos de contención de costos, como barreras económicas al acceso y a la utilización de servicios de salud.

\section{Agradecimientos}

Este proyecto fue desarrollado en la Escuela Superior de Administración Pública (ESAP), Programa Gerencia de Servicios de Salud, Bogota, Colombia, como parte del Programa Equity-oriented Health Policy Analysis in Latin America, implementado por la Red de Investigación en Sistemas y Servicios de Salud en el Cono Sur de América Latina, financiado por el Internacional Development Research Center, de Canadá. Agradecemos la asesoría brindada por Celia Almeida, Secretaria Ejecutiva de la Red y docente e investigadora de la Escuela Nacional de Salud Pública,
Fundación Oswaldo cruz (FIOCRUZ), y por Claudia Travassos, docente e investigadora del Centro de Información Científica y Tecnológica (CICT/FIOCRUZ), Rio de Janeiro, Brasil; el apoyo brindado por Camilo Uribe, director de los Postgrados en Gerencia de Servicios de Salud (ESAP), Colombia, y la asesoría brindada también por varios de sus docentes, incluidos Jairo Reynales y Rafael Martínez; y, finalmente, el apoyo proporcionado por un equipo de asistentes, incluidos Janeth Angarita, Edgar Garzon, Sonia Olaya y Fernando Basto. 


\section{Referencias}

AHUMADA, C., 1999. Reforma del Estado y políticas de salud en Colombia: Un enfoque de salud internacional. In: Políticas y Servicios Sociales Para el Siglo XXI (C. U. Mallarino, org.), pp. 279-292, Bogotá: Pontificia Universidad Javeriana.

ALAMES (Asociación Latinoamericana de Medicina Social), 2000. Declaración Política Final del VIII Congreso Latinoamericano de Medcina Social y del XI Congreso de la Asociación Internacional de Políticas deSalud. Manuscrito. La Habana: Grupo Estructura Salud. (mimeo.)

ALMEIDA, C., 1999. Reforma do Estado e reforma dos sistemas de saúde: Experiências internacionais e tendências de mudança. Ciencia \& Saúde Coletiva, 4:263-286.

ALVARADO, E., 1998. El Régimen Subsidiado de Salud. 1a Ed. Bogotá: Fundación Crecer.

BID (Banco Interamericano de Desarrollo), 1995. Acceso Equitativo a los Servicios Bási cos deSalud: Hacia una Agenda Regional para la Reforma del Sector Salud. Reunión especial sobre Reforma del Sector Salud. Washington, DC: BID.

BITRAN, R. \& CESPEDES, J. E., 2001. Resumen ejecutivo. In: Proyecto Análisis de los Problemas y Soluciones Relacionados con el Sistema deVigilancia y Control del Sector Salud (J. E. Cespedes, org.), pp. 22-26, Bogotá: Programa de Apoyo a la Reforma de Salud, Ministerio de Salud/Unión Temporal Bitrán y Asociados-Econometría.

BOSSERT, T.; LARRAÑAGA, O. \& RUIZ, F., 2000. Decentralization of health systems in Latin America. Revista Panamericana deSalud Pública, 8:84-92.

CASTAÑO, R. A., 1999. Medicina, Ética y Reformas a la Salud. Bogotá: Fundación Crecer.

CASTAÑO, R. A.; BERMUDEZ, P.; REY, J. A. \& ZAWADSKY, M. F., 1999. Equidad en el Financiamiento de los Servicios de Salud en Colombia: Impacto del Gasto de Bolsillo. Bogotá: Fundación Crecer. (mimeo.)

CESPEDES, J. E.; LONDOÑO, J. L.; ESCOBAR, M. L., 1995. La Carga de Enfermedad en Colombia. Bogotá: Ministerio de Salud.

CESPEDES, J. E., 1998a. Consideraciones sobre la viabilidad financiera del sistema de seguridad social en salud. Gerencia y Salud, 16:6-11.

CESPEDES, J. E., 1998b. Cómo consolidar la reforma de salud. Seguridad Social al Día, 1:6-9.

CESPEDES, J. E., 2001a. Atención Gerenciada delas Enfermedades de Alto Costo en el ISS: Problema, Causas y Opciones. Documento Técnico. Bogotá: Unidad de Investigaciones en Salud, Instituto de Seguros Sociales.

CHERNICHOVSKY, D., 1995. Health system reforms in industrialized democracies: An emerging paradigm. Milbank Quarterly, 73:339-372.

COLOMBIA, 1990. Ley 10. Bogotá: Congreso de la República.

COLOMBIA, 1993a. Ley 60. Bogotá: Congreso de la República.

COLOM BIA, 1993b. Ley 100. Bogotá: Congreso de la República.

COLOMBIA, 1999a. Ley 489. Bogotá: Congreso de la República.

COLOMBIA, 1999b. Ley 608. Bogotá: Congreso de la República.
CULYER, A. J. \& WAGSTAFF, A., 1993. Equity and equality in health and health care. Journal of Health Economics, 12:431-457.

DANIELS, N., 1996. Justice and Justification: Reflective Equilibrium in Theory and Practice. New York: Cambridge University Press.

DeMURO, P. R., 1995. The Financial Manager's Guide to Managed Care \& Integrated Delivery Systems. New York: McGraw-Hill.

FELDSTEIN, P. J., 1998. Health Care Economics. 5th Ed. New York: Delmar Publishers.

FERNANDEZ, G., 1996. El Nuevo Sistema de Salud en Colombia. Bogotá: Castillo Editores.

FOLLAND, S.; GOODMAN, A. \& STANO, M., 1993. The Economics of Health and Health Care. New York: MacMillan.

FRANCO, A., 2000. La Reforma dela Salud en Colombia. Bogotá: Grupo Estructura Salud. (mimeo.)

FRENK, J., 1988. Financing as an instrument of public policy. Bulletin of the Pan American Health Organization, 22:440-446.

FUCHS, V., 1998. Who Shall Live? Health Economics and Social Choice. London: World Scientific Publishing $\mathrm{Co}$.

GIRALDO, J. C., 2000. Primera Encuesta Nacional de Calidad en Salud Percibida por los Usuarios. Bogotá: Defensoría del Pueblo.

GWATKIN, R. D, 2001. The need for equity - Oriented health sector reforms. International Journal of Epidemiology, 30:720-723

HARVARD UNIVERSITY, 1993. International Conference on Health Sector Reform in Developing Countries: Issues for the 1990's. Durham: Harvard School of Public Health.

HARVARD UNIVERSITY, 1996. La reforma del Sector Salud en Colombia y el Plan Maestro de Implementación (InformedeConsultoría). Durham: Harvard School of Public Health.

HERNANDEZ, A.; VEGA, R. \& CARDONA, J., 1995. Criterios Metodológicos para la Medición de Ine quidades en Salud y en la Prestación de Servicios deSalud en Colombia. Tesis de Maestría, Bogotá: Facultad de Ciencias Económicas y Administrativas, Posgrado en Administración de Salud y Seguridad Social, Pontificia Universidad Javeriana.

HERNANDEZ, M., 2000. El enfoque sociopolítico para el análisis de las reformas sanitarias en América Latina. In: VIII Congreso Latinoamericano de Medicina Social y XI Congreso de la Asociación Internacional de Políticas de Salud. CD-ROM. La Habana: Asociación Latinoamericana de Medicina Social.

HERNANDEZ, P. O. \& ZAPATA, A. L., 1991. Equidad y salud: Necesidades de investigación para la formulación de una política social. Salud Pública de México, 33:9-17.

HOSMER, D. W. \& LAMESHOW, S., 1989. Applied Logistic Regression. New York: John Wiley \& Sons.

HSIAO, W. C., 1994. Marketization - The illusory magic pill. Health Economics, 3:351-357.

HSIAO, W. C., 1998. Financing Health Care in Developing Countries. Technical Document. Washington, DC: World Bank.

HSIAO, W. C. \& LIU, Y., 1996. Economic reform and 
health - lessons from China. New England Journal of Medicine, 335:430-432.

ISAACS, F., 2000. Aproximación al análisis del sistema general de seguridad social en salud desde la teoría de sistemas auto-referentes. In: Proyecto Análisis de los Problemas y Soluciones Relacionados con el Sistema deVigilancia y Control del Sector Salud (J. E. Céspedes, org.), pp. 2-10, Bogotá: Programa de Apoyo a la Reforma de Salud, Ministerio de Salud/Unión Temporal Bitrán y AsociadosEconometría.

JACK, W., 1999. Principles of Health Economics for Developing Countries. Washington, DC: World Bank.

JAMISON, D.; MOSLEY, M.; MEASHAM , A. \& BOBADILLA, J. L., 1993. Disease Control Priorities in Developing Countries. New York: Oxford University Press.

JARAMILLO, I., 1999. El Futuro dela Salud en Colombia. Ley 100 de 1993. Cinco años Después. 4a Ed. Bogotá: Fundación Corona/Fundación Friedrich Ebert de Colombia/Fundación para la Educación Superior/Fundación Restrepo Barco.

KAKWANI, N. C., 1977. Measurement of tax progressivity: An international comparison. Economic Journal, 87:71-80.

LONDOÑO, J. L. \& FRENK, J., 1997. Structured pluralism: Towards an innovative model for health system reform in Latin America. Health Policy, 41:136.

MALAGA, H.; LATORRE, M. C.; CARDENAS, J.; MONTIEL, H.; SAM PSON, C.; TABORDA, M. C.; GRANADOS, R. \& PASTTOR, D., 2000. Equidad y reforma en salud en Colombia. Revista deSalud Pública, 2:1-18.

MARSET-CAMPOS, J. M.; SAEZ-GOMEZ, J. M . \& SANCHEZ-MORENO, A., 1998. Análisis comparativo de los sistemas de salud. In: Salud Pública (F. Martinez-Navarro, org.), pp. 819-845, Madrid: McGraw-Hill Interamericana.

MOLINA, C. G.; RUEDA, M. C. \& VELEZ, C. E., 1993. Gasto Público en Salud y Distribución deSubsidios en Colombia. Bogotá: Departamento Nacional de Planeación, Banco Mundial.

MORALES, L. G., 1997. El Financiamiento del Sistema de Seguridad Social en Salud en Colombia. Serie Financiamiento del Desarrollo 55. Santiago: Comisión Económica para América Latina y el Caribe.

MURRAY, C. J. L., 1996. Rethinking DALYs. In: The Global Burden of Disease (C. J. L. Murray \& A. D. López, ed.), pp. 1-98, Cambridge: Harvard University Press.

NEUFELD, V. \&JOHNSON, N. (ed.), 2000. Forging Links for Health Research. Ottawa: International Development Research Centre.

NIETO-LOPEZ, E., 2000. Factores Soci oeconómi cos y Aseguramiento en Salud en el Área Urbana de CoIombia. Tesis de Maestría, Medellín: Facultad Nacional de Salud Pública.

PAHO (Pan American Health Organization)/WHO (World Health Organization), 2001. Equity \& Health: Views from the Pan American Sanitary Bureau. Washington, DC: Health and Human Development Division, PAHO.
PATIÑO, J. F. (ed.), 1999. Ley 100 de 1993: Reforma y Crisis dela Salud. Bogotá: Academia Nacional de Medicina.

PREKER, A.; HARDING, A. \& GIRISHANKAR, N., 1999. The Economics of Private Participation in Health Care: New Insights from Institutional Economics. Technical Document. Washington, DC: World Bank.

RAM ALINGASWAMI, V., 1993. Health research, a key to equity in health development. Social Science and Medicine, 36:103-108.

RESTREPO, M., 1997. La Reforma a la Seguridad Social en Salud en Colombia y la Teoría de la Competencia Regulada. Serie Financiamiento del Desarrollo 47. Santiago: Comisión Económica para América Latina y el Caribe.

REYES, A. \& CESPEDES, J. E., 1999. Evasión y Elusión de Aportes a la Seguridad Social en Salud en Santafé de Bogotá. Bogotá: Econometría/ Secretaría Distrital de Salud.

REYES, A.; CESPEDES, J. E. \& RAMIREZ, M., 1998. Análisis de las Encuestas de Calidad deVida para Evaluar el Impacto del Nuevo Sistema General de Seguridad Social en Salud. Bogotá: Econometría/ Departamento Nacional de Planeación.

RUIZ, F. \& O'M EARA, G. S., 1999. La eficiencia en el mercado regulado de servicios de salud. In: Políticas y Servicios Sociales Para el Siglo XXI (C. U . Mallarino, org.), pp. 225-251, Bogotá: Pontificia Universidad Javeriana.

SANCHEZ, F. \& MENDEZ, J., 1999. Descentralización, pobreza y acceso a los servicios sociales: ¿Quién se benefició del gasto público social en los noventas? In: Políticas y Servicios Sociales Para el Siglo XXI (C. U. Mallarino, org.), pp. 93-129, Bogotá: Pontificia Universidad Javeriana.

SARMIENTO, A., 1999. La equidad y los servicios sociales básicos en Colombia. In: Políticas y Servicios Sociales Para el Siglo XXI (C. U. Mallarino, org.), pp. 55-91, Bogotá: Pontificia Universidad Javeriana.

SEN, A., 1987. On Ethics \& Economics. Oxford: University of California.

SEN, A., 1992. Inequality Reexamined. Boston: Harvard University Press.

SEN, A., 1999. La Salud en el Desarrollo. Discurso inaugural pronunciado ante la 52a Asamblea Mundial de la Salud. 18 de mayo de 1999 ঝttp:// www. who.int.infwha52/to_our_health/amartya.html>

SPSS INCORPORATION, 1997. SPSS for Windows. Statistical Packagefor the Social Sciences. Release 8.0. Chicago: SPSS Inc.

STATA CORPORATION, 1999. Stata Statistical Software: Release 6.0. College Station: Stata Corporation.

TOWNSEND, P. \& DAVIDSON, N., 1995. The Black Re port: Inequalities in Health. Ontario: Penguin Books.

van DOORSLAER, E.; WAGSTAFF, A. \& RUTTE, F., 1993. Equity in the Delivery of Health Care: An International Perspective. Oxford: Oxford University Press.

VELEZ, C. E., 1994. La Incidencia del Gasto Público Social en Colombia. Bogotá: Departamento Nacional de Planeación

WAGSTAFF, A. \& van DOORSLAER, E., 1998. Inequali- 
ties in Health: Methods and Results for Jamaica. Washington, DC: Human Department, World Bank. WAGSTAFF, A.; van DOORSLAER, E. \& PACI, P., 1991a. Horizontal equity in the delivery of health care. Journal of Health Economics, 10:251-256.

WAGSTAFF, A.; van DOORSLAER, E. \& PACI, P., 1991b. On the measurement of horizontal inequity in the delivery of health care. Journal of Health Economics, 10:169-205.

WAGSTAFF, A.; van DOORSLAER, E.; CALONGE, S.; CHRISTIANSEN, T.; GERFIN, M.; GOTTSCHALK, P.; JANSSEN, R.; LACHAUD, C.; LEU, R. E. \& NOLAN, B., 1992. Equity in the finance of health care: Some international comparisons. Journal of Health Economics, 11:361-387.

WAGSTAFF, A.; van DOORSLAER, E.; van der BURG, H.; CALONGE, S.; CHRISTIANSEN, T.; CITONI, G.; GERDTHAM, U.-G.; GERFIN, M.; GROSS, L.; HÄKINEN, U.; JOHNSON, P.; JOHN, J.; KLAVUS, J.; LACHAUD, C.; LAURITSEN, J.; LEU, R.; NOLAN, B.; PERAN, E.; PEREIRA, J.; PROPPER, C.; PUFFER, F.; ROCHAIX, L.; RODRIGUEZ, M.; SCHELLHORN, M.; SUNDIBERG, G. \& WINKELHAKE, O., 1999. Equity in the finance of health care: Some further international comparisons. Journal of Health Economics, 18:263-290.

WB (World Bank), 1993. World Development Report: Investing in Health. Washington, DC: WB.

WB (World Bank), 1997. World Devel opment Report 1997: The Statein a Changing World. Washington, DC: WB.

WB (World Bank), 2001. TheFlagship Course on Health Sector Reform and Sustainable Financing. Washington, DC: WB.

WHITEHEAD, M., 1992. The concepts and principles of equity and health. International Journal of Health Services, 22:429-445.

YEPES, F. J., 1999. La Salud en Colombia. Documento General. Bogotá: Departamento Nacional de Planeación, Ministerio de Salud.

YEPES, F. J., 2000. Se desarrolla en Colombia una cultura de aseguramiento. Boletín Trimestral del Núcleo de Acopio y Difusión de Información y Iniciativas de Reforma en Salud, 2:3-4.

Recibido el 8 de febrero de 2001

Versión final presentada el 12 de diciembre de 2001

Aprobado el 20 de marzo de 2002 